\title{
Water Column Structure and Statistics of Denmark Strait Overflow Water Cyclones
}

\author{
Wilken-Jon von Appen \\ Massachusetts Institute of Technology - Woods Hole Oceanographic Institution Joint Program in \\ Oceanography, Woods Hole, Massachusetts, USA ${ }^{1}$ \\ Robert S. Pickart and Kenneth H. Brink \\ Department of Physical Oceanography, Woods Hole Oceanographic Institution, Woods Hole, Massachusetts, \\ $U S A$ \\ Thomas W. N. Haine \\ Earth and Planetary Sciences, Johns Hopkins University, Baltimore, Maryland, USA
}

\begin{abstract}
Data from seven moorings deployed across the East Greenland shelfbreak and slope $280 \mathrm{~km}$ downstream of Denmark Strait are used to investigate the characteristics and dynamics of Denmark Strait Overflow Water (DSOW) cyclones. On average, a cyclone passes the mooring array every other day near the $900 \mathrm{~m}$ isobath, dominating the variability of the boundary current system. There is considerable variation in both the frequency and location of the cyclones on the slope, but no apparent seasonality. Using the year-long data set from September 2007 to October 2008, we construct a composite DSOW cyclone that reveals the average scales of the features. The composite cyclone consists of a lens of dense overflow water on the bottom, up to $300 \mathrm{~m}$ thick, with cyclonic flow above the lens. The azimuthal flow is intensified in the middle and upper part of the water column and has the shape of a Gaussian eddy with a peak depth-mean speed of $0.22 \mathrm{~m} / \mathrm{s}$ at a radius of $7.8 \mathrm{~km}$. The lens is advected by the mean flow of $0.27 \mathrm{~m} / \mathrm{s}$ and self propagates at $0.45 \mathrm{~m} / \mathrm{s}$, consistent with the topographic Rossby wave speed and the Nof speed. The total translation velocity along the East Greenland slope is $0.72 \mathrm{~m} / \mathrm{s}$. The self-propagation speed exceeds the cyclonic swirl
\end{abstract}

\footnotetext{
${ }^{1}$ Now at Alfred Wegener Institute for Polar and Marine Research, Bremerhaven, Germany
} 
speed, indicating that the azimuthal flow cannot kinematically trap fluid in the water column above the lens. This implies that the dense water anomaly and the cyclonic swirl velocity are dynamically linked, in line with previous theory. Satellite sea surface temperature (SST) data are investigated to study the surface expression of the cyclones. Disturbances to the SST field are found to propagate less quickly than the in-situ DSOW cyclones, raising the possibility that the propagation of the SST signatures is not directly associated with the cyclones.

Keywords: Denmark Strait Overflow Water cyclone, East Greenland boundary current system, East Greenland Spill Jet, Deep Western Boundary Current

\section{Introduction}

The dense water passing southward through Denmark Strait comprises the largest contribution to the lower limb of the Atlantic meridional overturning circulation. As such, the mixing, entrainment, and precise pathways of the water south of the strait impact the global climate. While the mean equatorward flux of the dense water across the sill between Iceland and Greenland is fairly well estimated (Jochumsen et al., 2012), the detailed, time-dependent nature of the flow is not fully understood. The densest portion of the outflow from Denmark Strait (sill depth of $650 \mathrm{~m}$ ) is called Denmark Strait Overflow Water (DSOW). Although the transport of DSOW appears to be relatively stable on seasonal to interannual timescales (Dickson and Brown, 1994; Macrander et al., 2005), the flow exhibits striking mesoscale variability with large changes in density and velocity (Worthington, 1969) occurring over periods of a few days (e.g. Macrander et al., 2007; Haine, 2010). It is essential to understand how such high frequency variability of the Denmark Strait overflow impacts the fate of the dense water downstream of the strait.

During its initial descent from the sill, the Denmark Strait overflow accelerates and entrains ambient water thereby reducing its density (Price and O'Neil Baringer, 1994). However, this modified water still contributes to the densest component of the North Atlantic Preprint submitted to Deep Sea Research Part I: Oceanographic Research Papers 
Deep Water (NADW) which ventilates a significant portion of the deep World Ocean. As part of the adjustment process, the DSOW layer, as well as the overlying intermediate water, stretches vertically. Due to potential vorticity constraints this induces positive relative vorticity and leads to cyclone formation (Spall and Price, 1998). The dynamics associated with the propagation of a lens of dense water on a sloping bottom have been addressed in numerous studies.

The laboratory and numerical experiments of Whitehead et al. (1990) showed that a cyclonic circulation exists vertically offset above the lens. Nof (1983) studied dense water lenses on a sloping bottom that are associated with an anti-cyclonic flow around the lenses at the same depth as the lenses and showed that they propagate at a speed proportional to the density anomaly and the bottom slope. Swaters and Flierl (1991) developed a two layer model of an isolated eddy which was later extended to include a stratified upper layer (Poulin and Swaters, 1999). Such an isolated eddy does not depend upon far-field interactions to be balanced as its pressure anomaly vanishes away from the eddy. This condition has been formalized as the "Stern integral constraint" (Mory, 1985). If the interaction between the lower dense water layer and the overlying water column is significant, then the model of Swaters and Flierl (1991) predicts strong cyclonic flow in the upper layer and weak azimuthal speeds in the deep layer consistent with Whitehead et al. (1990). In this case, the propagation velocity of the lower layer lens is faster than the azimuthal speed in either layer, but is still consistent with the "Nof speed". On the East Greenland slope the propagating lenses of overflow water with overlying cyclonic circulation are called "DSOW cyclones". In the model of Spall and Price (1998) the cyclones form from a steady outflow; however, presently it is unknown how the time-dependent boluses of DSOW in the strait are related to this cyclogenesis process.

[Figure 1 about here.]

Girton et al. (2001) observed the initial formation and descent of the DSOW cyclones 
44 just south of the sill. Their subsequent evolution along the East Greenland slope was studied 45 with an idealized numerical model by Spall and Price (1998) and with realistically configured simulations by Käse et al. (2003) and Magaldi et al. (2011). However, observational studies 47 of the cyclones downstream of the strait have been limited to two surface-based studies. 48 Krauss (1996) used the tracks of surface drifters drogued at $100 \mathrm{~m}$ depth to identify and de-

scribe three cyclonic features that moved equatorward along the East Greenland slope. The measurements suggested a Gaussian eddy with a radius of $10 \mathrm{~km}$ that progressed southward due to self-propagation as well as advection by the background current. Bruce (1995) used satellite imagery to track disturbances ("hooks", "spirals", etc) to the sea surface temperature (SST) front between cold East Greenland Current water inshore of the shelfbreak and warm Irminger Current water offshore of the shelfbreak. Based on 33 observations, Bruce (1995) found that the features propagated southward at roughly $0.27 \mathrm{~m} / \mathrm{s}$ which is somewhat slower than what Krauss (1996) deduced. The inferred average radius was $17 \mathrm{~km}$. Bruce (1995) then compared the structures in SST with theoretical and laboratory studies (e.g. Whitehead et al., 1990) and argued that the SST disturbances are the surface signature of the DSOW cyclones.

The boundary current system along the East Greenland continental slope (schematically shown in Figure 1) consists of three distinct components in addition to the variable flow associated with the DSOW cyclones. The East Greenland/Irminger Current is a surfaceintensified flow supported by the horizontal density gradient between the Arctic-origin water on the shelf and the warm (denser) North Atlantic-origin water on the slope (e.g. Sutherland and Pickart, 2008; Brearley et al., 2012). The East Greenland Spill Jet (hereafter referred to as the spill jet) is a bottom-intensified flow on the upper slope that is comprised of dense waters that "spill" off the shelf south of Denmark Strait and subsequently adjust to form a southward-flowing current (Pickart et al., 2005; Harden et al., 2013). Finally, the Deep Western Boundary Current (DWBC) is the near-bottom equatorward flow that 

88 of Bruce (1995).

\section{Data}

\subsection{Mooring array}

transports the densest part of the NADW (Dickson and Brown, 1994). Interestingly, mooring observations east of Cape Farewell (the southern tip of Greenland) have found no sign of mesoscale variability similar to that of DSOW cyclones (Daniault et al., 2011; Bacon and Saunders, 2010). This suggests that the DSOW cyclones spin-down during their transit along the East Greenland slope.

The amount of entrainment of ambient water into the dense water overflow plume south of Denmark Strait determines the final properties of the newly formed NADW. As such, it is of high importance to understand and quantify the processes that dictate the evolution of the flow during its adjustment along the East Greenland continental slope. In an effort to learn more about the boundary current system in this region, a mooring array was deployed for a one-year period approximately $280 \mathrm{~km}$ southwest of Denmark Strait. The array extended from the outer-shelf to the deep slope and hence captured the East Greenland/Irminger Current, the spill jet, and the shoreward portion of the DWBC. It also sampled the frequent passage of DSOW cyclones which are the focus of this study. Using the mooring timeseries we first present the statistics of the eddies, and then construct a composite cyclone using the year-long data. In doing so we quantify the scales of the features, their downstream propagation, and the associated pressure field. Finally, using satellite imagery, we investigate the possible sea surface signature of the cyclones and compare this to the previous results

The mooring array on the East Greenland shelf and slope consisted of seven moorings deployed from 5 September 2007 to 4 October 2008. The moorings are labeled consecutively from EG1 (inshore-most mooring) to EG7 (offshore-most mooring). Their positions are shown in Figure 2, and Figure 3 details their configuration in the cross-stream plane. Details 
about the mooring array can be found in von Appen (2012). Here we briefly summarize the salient aspects of the array and the instruments used in this study.

[Figure 2 about here.]

[Figure 3 about here.]

Each of the moorings contained a conductivity-temperature-depth (CTD) profiler that nominally sampled twice a day from $100 \mathrm{~m}$ to just above the bottom. The four inshore moorings (EG1-4) employed Coastal Moored Profilers (CMPs) and the outer three moorings (EG5-7) used McLane Moored Profilers (MMPs; e.g. Morrison et al., 2000). Velocity was measured on the inner moorings using upward- and downward-facing acoustic Doppler current profilers (ADCPs) sampling hourly, and using travel-time acoustic current meters (ACMs) attached to the MMPs (measuring twice a day) on the outer moorings. As such, it was planned to obtain multiple vertical sections each day of hydrographic variables and velocity across the array. In addition, half hourly measurements of temperature, conductivity, and pressure were obtained from Microcats mounted near the ocean floor and on a $47 \mathrm{~m}$ long wire extending buoyantly above the top floats of the moorings.

Complications arose from the use of steel spheres for the top floats. In particular, the compasses of the ADCPs mounted on the floats were compromised by the magnetic fields induced in the spheres' steel by the Earth's magnetic field. Fortunately, the measurement range of the deep upward-facing ADCPs extended close to the depth of the top floats on the inner moorings, while the ACMs also measured velocities near the top floats on the outer moorings. This allowed for a direct comparison of the upper ADCP records to those of the deeper instruments near a common depth. The speed comparison was very favorable, while the angles differed as a function of the orientation of the flow. Using theory developed to correct compasses on steel ships (National Geospatial-Intelligence Agency, 2004), together with the angles measured by the deeper instruments, we were able to correct the 
compromised $\mathrm{ADCP}$ records to within $\approx 10^{\circ}$ of the current direction (see von Appen, 2012, for details).

Unexpectedly strong currents occurred regularly during the deployment period, associated with the passage of the DSOW cyclones. Velocities regularly exceeded $1 \mathrm{~m} / \mathrm{s}$ over large parts of the water column near the center of the eddies, while speeds of $1.5 \mathrm{~m} / \mathrm{s}$ were not uncommon. This resulted in mooring blowdowns that at times exceeded $500 \mathrm{~m}$ as determined from the pressure sensors on the microcats. During the blowdowns the moored profilers were either unable to traverse the wire (instead providing a point measurement at the location where the instrument was stuck) or returned a partial profile. In addition, the bottom mounted ADCPs tilted beyond the range of their tilt sensors $\left(23^{\circ}\right)$ during the larger blowdown events. Although the pressure sensors on the top microcats went beyond their rated range during the blowdowns, the resulting pressure records could be corrected as long as the depth was less than about $520 \mathrm{~m}$. Likely because of this extraordinarily energetic environment, all of the the CMPs stopped profiling prematurely. The shortest record was one month at mooring EG2, while the longest record was 8 months at mooring EG1 (although after the CMPs stopped profiling they returned point timeseries of temperature and salinity). Consequently, it was impossible to construct vertical sections as had been planned. However, the data return (particularly for the velocity) was sufficient to carry out our analysis of the DSOW cyclones.

The tidal signal on the outer shelf and upper slope (moorings EG1-3) was significant, with a combined amplitude for the constituents $\mathrm{O}_{1}(25.82 \mathrm{~h}), \mathrm{K}_{1}(23.93 \mathrm{~h}), \mathrm{M}_{2}(12.42 \mathrm{~h})$, and $\mathrm{S}_{2}(12.00 \mathrm{~h})$ of up to $25 \%$ of the standard deviation of the full velocity records. These tidal signals were removed from the ADCP records using a tidal fit to the data (Pawlowicz et al., 2002). Tidal amplitudes at the remaining moorings were less than $0.03 \mathrm{~m} / \mathrm{s}$ and therefore negligible compared to the typical variability in the records. Hence, the records at EG4-7 were not de-tided. After de-tiding, the velocities were rotated into an alongstream 
and cross-stream coordinate system. All obtained velocity records were combined into a single record and the alongstream direction was defined as the principle axis of variance of this record (the principal axis differed by less than $10^{\circ}$ for the different depths and different locations across the array). The resulting alongstream direction of $-110^{\circ} \mathrm{T}$ (i.e. west-southwestward) also coincides with the average direction of the shelfbreak topography in the study region (Figure 2). From here on, the variable $x$ denotes alongstream distance (positive equatorward), the variable $y$ denotes cross-slope distance (positive offshore), and the variable $z$ denotes vertical distance (positive upwards).

\subsection{Satellite SST data}

Satellite sea surface temperature (SST) images are used in this study. They are Level 2 products of the MODIS Aqua and MODIS Terra satellites. MODIS is the Earth Observing System (EOS) Moderate Resolution Imaging Spectrometer, and the processing steps for the Level 2 product are documented in Brown and Minnett (1999). The spatial resolution of the infrared satellite measurements at nadir is $1 \mathrm{~km}$, and the Level 2 product takes advantage of this full resolution without smoothing in space and time. The study region is cloud covered 80-90\% of the time. Since infrared radiation does not penetrate clouds, consecutive images are often several days apart. The Level 2 product contains a preliminary bad data detection flag. This captures both possible clouds and data pixels with temperatures strongly different from their surrounding pixels. Unfortunately, this tends to reject pixels near the high SST gradient region of the East Greenland /Irminger Current hydrographic front (where temperatures can range from from $0-2^{\circ} \mathrm{C}$ on the shelf to $8-10^{\circ} \mathrm{C}$ over the slope). Since this is a region of particular interest, we devised an adjusted cloud cover rejection routine as follows. Cloud tops are much colder than $-2^{\circ} \mathrm{C}$, the coldest reasonable ice-free SST. Hence, scattered clouds result in spots of unrealistically cold satellite-measured temperatures surrounded by a region of transitional temperatures where both sea-surface and cloud-top emitted infrared radiation reaches the spectrometer. Therefore, areas characterized by occurrences of these 
very cold temperatures were manually removed, leaving mostly continuous regions of temperatures in the $-2-12^{\circ} \mathrm{C}$ range, reasonable for ice-free SST. No further adjustments, other than removal of entire regions of the domain with questionable data, were applied.

\section{Methods}

As discussed above, when DSOW cyclones passed the array the mooring located near the cyclone center was significantly blown down (neighboring moorings were affected as well, although not as severely). As such, no complete hydrographic profiles were obtained in the center of the cyclones, only on the edges. Keep in mind, however, that the bottom microcats recorded temperature and salinity throughout. Regarding velocity, the ADCPs mounted on the top floats did record data during blowdowns, although their measurement depth was deeper than the intended 0-100 m range. In addition, the downward-facing ADCP mounted below the top float of mooring EG4 (bottom depth of $900 \mathrm{~m}$ ) was functional during these events. Consequently, we did obtain velocity profiles at both the center and the edges of the cyclones. Fortuitously (as detailed below), the majority of the cyclones passed near mooring EG4. At this location a nearly continuous ( $>95 \%$ of planned measurements) timeseries of velocity was recorded spanning the middle portion of the water column (from $260 \mathrm{~m}$ to $660 \mathrm{~m}$ depth). Hence, despite the data gaps, there was enough information to provide a detailed view of the velocity structure of the cyclones, with complementary hydrographic information near the sides of the features as well as along the bottom.

[Figure 4 about here.]

As an example of how these cyclone passages are recorded by the mooring array, Figure 4 presents the velocity records obtained during the passage of a DSOW cyclone onshore of mooring EG6. As the cyclone passed near EG6 (at 0930Z on October 19, 2007), the cross-stream velocity changed from strongly offshore to strongly onshore (Figure 4f,h,j). At 
EG6 (near the eddy center), the mooring blowdown temporarily lessened at this time (Figure $4 \mathrm{~g}, \mathrm{~h})$. On the onshore side, the eddy swirl velocity led to an increase in the downstream flow (Figure 4c,e) that did not extend to EG3 on the upper slope (Figure 4a). Conversely, on the offshore side, the swirl velocity of the cyclone led to a decrease in the alongstream velocity (Figure 4i).

This sequence of events was qualitatively the same for all passing cyclones. As such, we developed a methodology within the framework of a graphical user interface to identify when and where DSOW cyclones passed the mooring array. This encompassed only the moorings on the continental slope, EG3-7 (no cyclones were detected on the shelf). The timeseries in question were visually inspected in two-day segments (similar to Figure 4) and the time of passage of a given DSOW cyclone was identified as a continuous variable, while its cross-slope location was identified as a discrete variable that could take 15 distinct bin values: onshore of EG3, near EG3, offshore of EG3, onshore of EG4, near EG4, etc. The fact that the number of eddies identified within the two bounding bins (onshore of EG3 and offshore of EG7) accounts for less than $5 \%$ of all identified eddies (Figure 6 below), suggests that these discrete bins essentially bracket the locations at which DSOW cyclones pass the mooring array.

[Figure 5 about here.]

The velocity field measured by the moorings during the passage of a cyclone is schematically shown in Figure 5. Comparing this schematic to examples such as the one shown in Figure 4, the following criteria were devised for eddy detection. The first two criteria are required for the identification of a cyclone, while the remaining three criteria provide supporting information:

1. The cross-stream velocity switches from strongly offshore to strongly onshore. The time of the eddy passage corresponds to when this transition occurs at the mooring 
2. The downstream (positive alongstream) velocity increases significantly. Such an increase is indicative that the mooring in question is located near or onshore of the eddy center.

3. The downstream (positive alongstream) velocity decreases at a mooring signifying that the mooring is offshore of the eddy center.

4. Mooring blowdown is a proxy for water column-integrated speed. Two successive vertical excursions of a mooring (with a partial recovery in between, e.g. Figure $4 \mathrm{~g}, \mathrm{~h}$ ) indicate that the mooring in question is near the center of the eddy, i.e. inside the radius of approximate solid body rotation where the azimuthal velocity decreases toward the center of the feature. A single vertical excursion, on the other hand, indicates that the respective mooring is near the edge of the eddy.

5. An increase and subsequent decrease in near-bottom potential density (or corresponding signature in potential temperature) at a mooring indicates the close proximity of a cyclone (i.e. the presence of DSOW). This information helped constrain both the time of passage of the cyclone as well as its cross-stream location.

Applying these criteria to the mooring data made it possible to unequivocally identify the cyclones. There were virtually no cases when only small amplitude variations in velocity consistent with the first two criteria were observed. Once the anomalies were larger than the background, they were typically strong (amplitudes larger than three times the values common in the absence of the cyclones) and also exhibited some or all of the three supporting criteria. Employing the five criteria made it possible to unambiguously assign roughly $50 \%$ of the identified eddies to a single horizontal bin. For the remaining cases, the placement into two neighboring bins was ambiguous and the final assignment to one of those bins was done subjectively, which should be considered as part of the uncertainty in the resulting locations of the eddies as determined from this procedure. Based on the distance between 
the moorings (which increases from $7 \mathrm{~km}$ on the upper slope to $10 \mathrm{~km}$ in deeper water), the cross-stream locations of the cyclones are known to within $2-3 \mathrm{~km}$. Based on the sampling rate of the ADCPs, the times when the cyclones passed the array are known to within 1 hour.

\section{Cyclone Statistics}

In the 395 days of mooring array data, 190 cyclonic eddies were identified using the method described above. A histogram of their occurrence in the cross-stream plane is shown in Figure 6 (blue bars). Because the widths of the bins change across the slope, we also show a normalized histogram (red curve) which indicates the number of eddies per $2 \mathrm{~km}$ of cross-slope distance observed over a year. One sees that the cross-stream distribution of the cyclones is strongly peaked approximately $10 \mathrm{~km}$ seaward of the shelfbreak in the vicinity of mooring EG4 at a water depth of $900 \mathrm{~m}$ (Figure 6). This implies that more than 60 cyclones per year pass by this location. Note that the eddy count decreases sharply in the onshore direction, consistent with the notion that there are no cyclones at or inshore of the shelfbreak. The distribution decreases less rapidly in the seaward direction, and there are still eddy occurrences $40 \mathrm{~km}$ offshore of the shelfbreak near mooring EG7 in $1600 \mathrm{~m}$ water depth. However, the shape of the distribution suggests that the population of eddies offshore of the last bin is very small. The sill depth at Denmark Strait is $650 \mathrm{~m}$, hence the majority of the cyclones descend approximately $250 \mathrm{~m}$ over the $280 \mathrm{~km}$ distance to the mooring array. This corresponds to a vortex stretching of $40 \%$, which would lead to the generation of relative vorticity of $0.4 f$ ( $40 \%$ of the planetary vorticity) in the absence of frictional effects.

\section{[Figure 6 about here.]}

Is there temporal variability associated with this cross-stream distribution? The data indicate that while there are short-term trends, as happens for the running mean of any 
random function, there are no apparent longer term patterns (Figure 7a). In particular, there is no apparent seasonal signal. The separation time between consecutive eddies (Figure 7b) ranges from near zero to 8 days, with a mean of 2.1 days, although the two extreme separation times are rare. Near zero separation times occur when one cyclone passes close to the shelfbreak (near EG3) while another cyclone simultaneously passes far offshore (near EG7). The other extreme corresponds to extended periods with no cyclones at all during which the velocity variability was weaker than during periods with cyclones. As with the cross-stream distribution of the cyclones, the cyclone separation timeseries (Figure $7 \mathrm{~b}$ ) does not exhibit any longer term trends and no apparent seasonality. This is notable because the atmospheric forcing in this region does have a large seasonal signal, with strong winds and significant buoyancy forcing in the fall and winter months (e.g. Harden et al., 2011; Moore et al., 2013). This implies that the cyclones are not influenced by the atmosphere, and that the dynamics of their formation and propagation are a purely oceanic phenomenon. It is also consistent with the model results of Haine et al. (2009) and Spall and Price (1998), where DSOW cyclones form from a steady outflow through Denmark Strait.

[Figure 7 about here.]

On average, an eddy passes by the array every other day which means that they are a ubiquitous feature of the flow in this region. We note that the separation time of 2.1 days is very close to the 2.3 days computed by Bruce (1995) using SST data. However, the separation time in that study was calculated as the distance between consecutive eddies $(54 \mathrm{~km})$ divided by the translational speed $(0.27 \mathrm{~m} / \mathrm{s})$. As will be shown in the following sections, the method employed by Bruce (1995) appears to significantly underestimate the in-situ translational speed of the DSOW cyclones and, therefore, the agreement between the two separation time estimates could be coincidental. 


\section{Composite Cyclone}

As mentioned in Section 3, the only complete velocity timeseries returned by the array is in the middle water column at mooring EG4. Fortuitously, the vast majority of DSOW cyclones passed the array in the vicinity of this mooring (Figure 6). We now describe a statistical method that maps out the full three dimensional velocity structure of a composite DSOW cyclone using only velocity data from mooring EG4.

The data at EG4 capture different parts of the passing cyclones depending on the proximity of the cyclones to the mooring. For example, EG4 records the velocity on the offshore edges of eddies passing at EG3, and it records the velocity near the centers of eddies passing at EG4. If the eddies passing the different locations are statistically similar, then their mean structure can be determined in the following way. The measurements at EG4 during the 17 times when eddies passed at EG3 map out the offshore edge of the mean eddy. Likewise, the measurements at EG4 during the 33 times when eddies passed at EG4 map out the center of the mean eddy. While eddies passing at different depths are going to be somewhat different (e.g. in the degree of their stretching), for the following analysis we assume that the property variation in the cross-stream direction is small over the diameter of the eddies. An investigation of the degree of cross-stream variation (von Appen, 2012) supports this assumption, as do the results below.

We now composite the Eulerian mean structure of DSOW cyclones in the vicinity of mooring EG4 starting with the depth-mean velocity field between $260 \mathrm{~m}$ and $660 \mathrm{~m}$, where the velocity measurements are complete. Later in the paper (Subsection 5.5) we examine the vertical structure of the typical cyclone. Although one may wonder how representative this composite eddy is, our data are unfortunately not able to objectively quantify this. We note, however, that the velocity expressions of many of the cyclones as seen in the graphical user interface were qualitatively and quantitatively similar. This implies that the scales of the composite cyclone as described here are in fact representative of a significant number of 
the individual cyclones that passed the mooring array.

\subsection{Depth-mean background velocity}

Figure 8 shows the depth-mean velocity field between $260 \mathrm{~m}$ and $660 \mathrm{~m}$ in a top-down view, with the center of the cyclone located at $(x=0, y=0)$. The observer is situated on the East Greenland shelf looking offshore, so that the mean flow and the cyclone translation are towards the right. Bins in the cross-stream direction are averages from all the eddies that passed at that particular offset from EG4. The temporal offset with respect to the time when the eddy passed the mooring array is indicated along the top of the plot with negative values corresponding to times preceding the arrival of the eddy center. We used the propagation velocity of the eddy (which we define and derive below) to transform the temporal measurements into alongstream distance, plotted along the bottom of the horizontal axis. Color indicates the depth-mean speed in each bin and the black lines indicate the vector velocity. The white line near $-11 \mathrm{~km}$ corresponds to the approximate location of the shelfbreak in this transformed coordinate system.

[Figure 8 about here.]

We now decompose the full velocity field $(u, v)$ as a function of alongstream and crossstream location into the following components, plus a residual encompassing noise as well as components that we cannot determine from the available data.

$$
\begin{array}{ll}
u(x, y)=u_{b}+u_{c}(x) & -v_{a}(r) \sin (\theta)+\text { residual } \\
v(x, y)=v_{b} & +v_{a}(r) \cos (\theta)+\text { residual }
\end{array}
$$

Here $r=\sqrt{x^{2}+y^{2}}$ is the radius from the center and $\theta=\arctan \left(\frac{y}{x}\right)$ is the azimuthal angle measured counterclockwise with $0^{\circ}$ being in the direction of the mean flow. The first component is the background mean flow that is also present in the absence of DSOW 
cyclones. The available data allow us to estimate the background velocity $\left(u_{b}, v_{b}\right)$ whose alongstream and cross-stream components are uniform in space. The next component is the velocity with which the cyclone self-propagates. As the cyclone propagates along the East Greenland slope, it induces a velocity in the ambient fluid (including in the wake of the cyclone). These two velocity components have alongstream and cross-stream structure, but with the available data we are only able to determine the alongstream structure of the sum of these two components. We call this sum, which includes the translation of the cyclone and the induced velocity in the ambient water, the "co-translational" velocity $u_{c}(x)$. This means that the cross-stream component and structure of the co-translational velocity field is contained in the residual which we cannot determine from the available data. We note that this co-translational velocity is the Eulerian velocity measured by the moorings as the cyclones pass the array. It will be weak far away from the cyclones where the influence of the features is weak. The co-translational velocity $u_{c}(x)$ is also different from the spatially uniform propagation velocity of the frame of reference in which the cyclone dynamics can be evaluated. The frame of reference propagates with the total velocity at the exact center of the eddy (see Lilly and Rhines, 2002) which in our notation is $u_{b}+u_{c}(x=0)$. Finally, we determine the azimuthal velocity $v_{a}(r)$. All of the above velocity components are depth-mean quantities.

There is significant flow in the absence of cyclones, associated with the East Greenland/Irminger Current, the spill jet, and the DWBC. The influence of a DSOW cyclone persists for less than 18 hours before and after its center passage (Figure 8). Roughly 140 days (35\% of the velocity record) are more than 18 hours away from the center of a cyclone passing the mooring array. The depth-mean background flow in the alongstream direction during those 140 days is $u_{b}=0.27 \mathrm{~m} / \mathrm{s}$ equatorward and $v_{b}=0.04 \mathrm{~m} / \mathrm{s}$ directed offshore (Figure 8b). Given the angular uncertainty in the current direction (compare the definition of the alongstream direction), this offshore velocity is not meaningfully different 
from zero.

\subsection{Depth-mean flow associated with the translation of the composite cyclone}

[Figure 9 about here.]

Subtracting $\left(u_{b}, v_{b}\right)$ from the full flow field reveals the velocity structure of the eddy with respect to the background flow (Figure 9). Away from the feature there is very weak flow with essentially no structure. On the onshore side of the cyclone (negative cross-stream distance), the positive co-translational velocity and the positive azimuthal velocity superpose resulting in strong downstream velocity. On the offshore side, the positive co-translational velocity and the negative azimuthal velocity result in weak downstream flow. Along the center slice of the cyclone $(y=0)$, the alongstream flow is due only to the translation of the fluid with the cyclone and to the motion that is induced in the ambient fluid that is affected by the passing cyclone. In particular, the azimuthal velocity of the eddy does not contribute to the co-translational velocity $u_{c}(x)$ along $y=0$. The same is true for the cross-stream average over an area that is symmetric around $y=0$. In order to decrease the noise in the estimate of $u_{c}(x)$, we averaged the velocities in each bin between $y=-6 \mathrm{~km}$ and $y=6 \mathrm{~km}$ at each alongstream location to obtain the profile of the co-translational velocity (Figure 9b).

The co-translational velocity increases from approximately zero before the cyclone, reaching a maximum of $0.45 \mathrm{~m} / \mathrm{s}$ about $2.5 \mathrm{~km}$ after of the center of the cyclone, and decreases thereafter (but not back to zero). We assume that this maximum value approximately corresponds to the translational velocity $u_{t}$ of the cyclone with respect to the background flow. This is consistent with the model of an isolated self-advecting eddy that could be envisioned as a vertical cylinder being dragged through a fluid. The alongstream flow is due to the translation of the circularly symmetric feature and to the induced motion in the ambient fluid. This induced motion has a component that is symmetric before and after the translating feature and also contains the wake, which is only present on the trailing side. Due to 
the velocity in the wake, the alongstream velocities behind the cyclone are stronger than in front of it. Since DSOW cyclones are not characterized by step discontinuities in properties (as a dragged cylinder would be), the co-translational velocity ramps up to and down from its center value in a smooth fashion, as seen in Figure 9. The derivation of the precise detailed structure of $u_{c}(x)$ for a baroclinic Gaussian eddy on a topographic $\beta$-plane with stratification is complicated and beyond the scope of the present work. We note, however, that the qualitative elements of $u_{c}(x)$ deduced here correspond to the expected structure: near-zero velocity far from the cyclone, nearly constant translational velocity in the small (approximate solid body) core of the cyclone, and a smooth ramp up/down of the induced motion in the domain of influence of the cyclone in the ambient fluid.

Self-advection of a DSOW cyclone is consistent with the propagation of a finite amplitude, non-linear topographic Rossby wave. The restoring force for a topographic Rossby wave is associated with the change in potential vorticity experienced as the vortex column migrates into deeper or shallower water. For long waves, the linear topographic Rossby wave speed is (Pedlosky, 2003):

$$
c=-\beta R_{d}^{2}=\frac{f}{H_{0}} \frac{d H}{d y} R_{d}^{2},
$$

where $R_{d}$ is the internal Rossby radius, $\beta=\frac{-f}{H_{0}} \frac{d H}{d y}$ is topographic $\beta, f$ is the Coriolis parameter, and $H_{0}$ is the mean water depth of the isobath along which the topographic Rossby wave propagates. To estimate this speed, we take $H_{0}$ (here considered as a positive quantity) as the bottom depth of EG4 (900 m), and approximate the bottom slope as the difference in water depth between EG3 and EG5 (650 m) divided by their separation (14 km). The stratification $N$ in the middle water column is $2.2^{*} 10^{-3} \mathrm{~s}^{-1}$ (Figure $13 \mathrm{c}$ below). Different estimates for the Rossby radius are common: $R_{d}=\frac{N H}{f}$ and $R_{d}=\frac{N H}{\pi f}$, leading to a range of 5-15 km at $900 \mathrm{~m}$. The approximate radius of the cyclones of $8 \mathrm{~km}$ (Subsection 5.3 below) is within this range. Taking $R_{d}$ to be $8 \mathrm{~km}$ results in a topographic Rossby wave speed 
of $0.44 \mathrm{~m} / \mathrm{s}$, very similar to our observational estimate of the self-advection velocity with respect to the background flow $u_{t}(0.45 \mathrm{~m} / \mathrm{s})$. We note that there is significant uncertainty in this estimate due to the wide range in and the squared dependence on the Rossby radius, and there will be a correction factor due to the difference between linear wave dynamics and the finite amplitude vortex dynamics of the DSOW cyclones. Another way to predict the propagation velocity is $c=\frac{g^{\prime}}{f} \frac{d H}{d y}$ as proposed by Nof (1983) where $g^{\prime}$ is the reduced gravity $\frac{\Delta \rho}{\rho_{0}} g$. The density anomaly $\Delta \rho$ is not well-defined for our continuous stratification situation. However, assuming reasonable values $\left(\approx 0.1 \mathrm{~kg} / \mathrm{m}^{3}\right)$ for the density anomaly results in the same order of magnitude for the propagation speed. As such, the good agreement between the predicted and observed value of $u_{t}$ suggests that the restoring force associated with the deflection of a vortex column can account for the observed self-advection.

The sum of the translational velocity with respect to the mean flow $u_{t}$ and the background mean velocity $u_{b}$ is the speed of the cyclone with respect to the bottom. It reaches a maximum of $0.72 \mathrm{~m} / \mathrm{s}$ (Figure $9 \mathrm{~b}$ ). The sum of the background velocity $u_{b}$ and the cotranslational velocity $u_{c}(x)$ is the speed of a fluid parcel with respect to the bottom and this is the speed that was used earlier to transform the time axis into alongstream distance.

\subsection{Depth-mean azimuthal flow of the composite cyclone}

[Figure 10 about here.]

Next we remove both the background mean flow and the co-translational velocity to reveal the cyclonic flow of the isolated eddy (Figure 10). One sees that there is no flow at the center and that it increases and then decreases with radius. The concentric circles shown in Figure 10a are for visual guidance. It is clear that the majority of the flow is tangential to these circles as expected for an azimuthal flow.

[Figure 11 about here.] 
Plotted as a function of radius, the binned azimuthal velocities nicely reveal the structure of the composite eddy (Figure 11a). While there is obvious scatter, a smoothed $1 \mathrm{~km}$ running mean of the azimuthal velocities shows a very clear signal. Starting from zero, the azimuthal speed increases nearly linearly, reaches a maximum near $6 \mathrm{~km}$ and then decreases smoothly to near zero around 25-30 km. The most common model for a vortex is a simple Rankine vortex which exhibits azimuthal speed proportional to radius up to a distance and then inversely proportional to radius (and results from an isolated step discontinuity in potential vorticity which is a good theoretical model, but is not expected to occur in reality). A Rankine vortex fits the linear increase due to solid body rotation near the center, but is not a good model farther out as it does not decrease quickly enough compared with the data in Figure 11a. In contrast, a Gaussian eddy (which results from a smooth PV anomaly) is an excellent model (Figure 11a). The structure of a Gaussian eddy is given by:

$$
v_{a}(r)=v_{0} \frac{r}{R_{0}} e^{\frac{1}{2}\left(1-\left(\frac{r}{R_{0}}\right)^{2}\right)} .
$$

The azimuthal velocity increases nearly linearly up to a radius $R_{0}$ where it smoothly reaches its maximum velocity $v_{0}$. Beyond this radius the velocity decreases proportional to $e^{-r^{2}}$. Unlike the Rankine vortex, the influence of the Gaussian eddy is well bounded. The fit shown in Figure 11a gives an eddy radius of $R_{0}=7.8 \mathrm{~km}$ and a peak azimuthal depth-mean velocity of $v_{0}=0.22 \mathrm{~m} / \mathrm{s}$. We also note that the average profile in Figure 11a cannot be explained as the composite of many Rankine vortices with randomly varying parameters $\left(R_{0}\right.$ and $\left.v_{0}\right)$. Such a composite of Rankine vortices may have a similar shape to the data distribution near $R_{0}$, but, at radii $>1.5 R_{0}$, the velocity only decreases as $\frac{1}{r}$. This is in contrast to the much steeper decay of $e^{-r^{2}}$ (Gaussian eddy) seen in Figure 11a. This velocity fit is used below (Subsection 5.6) to infer the pressure field associated with DSOW cyclones. 
The Rossby number $\epsilon=\frac{v_{0}}{f \cdot R_{0}}$ for these fitted parameters at $r=R_{0}$ is $\approx 0.22$, which indicates that, in the mean, these eddies are nearly geostrophic, but ageostrophic effects are important. It should also be noted that the descent from $650 \mathrm{~m}$ at the Denmark Strait sill to $900 \mathrm{~m}$ at mooring EG4 leads to a vortex stretching of $250 \mathrm{~m}$ or $\approx 40 \%$. If the flow is barotropic, in the absence of friction this predicts a relative vorticity of $\zeta=0.4 f$ and a Rossby number $\epsilon=\frac{\zeta}{f}$ of 0.4 . Considering that the flow is not strictly barotropic and that frictional effects lead to some loss of relative vorticity, the observed cyclones are consistent with having been generated by vortex stretching. The depth-mean azimuthal velocity fit is also shown in Figure 10b.

The estimates of the translational velocity $u_{b}+u_{t}=0.72 \mathrm{~m} / \mathrm{s}$ and the radius of the cyclones $R_{0}=7.8 \mathrm{~km}$ given above depend on our velocity decomposition as defined in Equations (1) and (2). They differ significantly from the previous estimates of these quantities presented by Bruce (1995), $0.27 \mathrm{~m} / \mathrm{s}$ and $17 \mathrm{~km}$, respectively, based on sea surface temperature data. Possible explanations for this difference are discussed in Section 6 below. We now present a separate argument which does not rely on the velocity decomposition to support the notion that DSOW cyclones are comparatively small and fast. It is assumed that the eddies are approximately circular and not, for example, elongated in the alongstream direction. The radius of maximum azimuthal velocity is where a mooring experiences its greatest blowdown. As seen in the example of Figure 4, the passage of a cyclone over a mooring leads to a double-dip blowdown in the timeseries data. If the mooring was near the radius of maximum azimuthal velocity, this double-dip would not be pronounced as the intermediate recovery would be very short compared to the hourly measurement interval. Only during a very small number of cyclones was a distinct and well-defined double-dip observed at more than one mooring. This suggests that the diameter of most cyclones is somewhat smaller than about twice the average cross-stream mooring spacing of $8 \mathrm{~km}$. Hence their radius of maximum velocity is somewhat smaller than $8 \mathrm{~km}$ as determined from the ADCP and 
pressure sensor data on all moorings and consistent with the above estimate of $R_{0}=7.8 \mathrm{~km}$. The typical scale of 5-8 $\mathrm{km}$ for the radius of maximum azimuthal velocity can also be seen in Figures $8 \mathrm{a}$ and 9 where the maximum velocities during the cyclone center passage $(t=0)$ is found at a cross-stream distance of 5-8 km.

The passage of the radius of maximum azimuthal velocity at $0 \mathrm{~km}$ cross-stream distance happens roughly $t_{0}=3$ hours before and after the cyclone center (see the temporal axis on top of Figure 10a). The majority of the eddy influence falls within twice that radius and about \pm 6 hours (Figures 9a and $8 \mathrm{a}$ ). This leads to a propagation speed of approximately $\frac{4 R_{0}}{4 t_{0}}=0.72 \mathrm{~m} / \mathrm{s}$ in very good agreement with our other estimate.

After removing the background flow, the co-translational velocity, and the azimuthal velocity of the cyclone, the residual velocities (not shown) are weak (less than $0.1 \mathrm{~m} / \mathrm{s}$ ). This demonstrates that we have successfully decomposed the depth-mean velocity field associated with DSOW cyclones into the components shown in Figure 10b. Interestingly, there is an indication for increased offshore flow in the region onshore and in front of the cyclone (Figure 10a). This is consistent with "Type II" spilling as described by Magaldi et al. (2011) where such DSOW cyclones draw dense fluid off the shelf that feeds the spill jet (and hence does not return on the trailing edge of the cyclone). We note, however, that the magnitude of this signal is fairly weak compared to the noise level of our method.

\subsection{Bottom density associated with the composite cyclone}

[Figure 12 about here.]

Mooring EG4 was also equipped with a microcat that measured temperature and salinity near the bottom. Within a radius of about $5 \mathrm{~km}$ the bottom potential density (referenced to the surface) exceeds $27.8 \mathrm{~kg} / \mathrm{m}^{3}$ in the composite cyclone (Figure 12). This indicates the presence of DSOW (as defined by Dickson and Brown (1994)) in the core of the cyclones. When plotted as a function of radius (Figure 11b), the bottom density anomaly displays a 
clear signature that is well approximated by the Gaussian fit

$$
\sigma_{e}^{\prime}(r)=\sigma_{0} e^{-\frac{1}{2}\left(\frac{r}{R_{0}}\right)^{2}}
$$

where the radius $R_{0}=7.8 \mathrm{~km}$ is the same as for the Gaussian eddy velocity fit of Equation (4) and the maximum density anomaly at the origin is $\sigma_{0}=0.073 \mathrm{~kg} / \mathrm{m}^{3}$.

It is important to note that while overflow water is present at $900 \mathrm{~m}$ bottom depth (depth of EG4) when the cyclone passes, water this dense is only found deeper than $\approx 1100 \mathrm{~m}$ in the background field. We argue that there is dense fluid inside the cyclone that is propagating at the same speed as the cyclone, and that there is a dynamic link between the azimuthal flow field and the density anomaly. This is different than what would be expected for the passage of a linear topographic Rossby wave. Note that the passage of both a linear wave and a cyclone starts with offshore directed velocities. In the former case this would bring lighter fluid from higher up on the continental slope to the depth of EG4, though we note that this argument would only hold exactly if density was a passive tracer. On the trailing edge, the onshore velocities would advect denser fluid up to the depth of EG4. That means that the decrease in near-bottom density expected from a wave is inconsistent with the observed increase in density in the center of the composite cyclone. However, for dense fluid to be kinematically trapped and advected by the cyclonic velocity field, the maximum azimuthal velocity must be greater than the translational velocity (e.g. Flierl, 1981). This is not the case for our observed peak azimuthal velocity of $0.22 \mathrm{~m} / \mathrm{s}$ and translational velocity of $0.45 \mathrm{~m} / \mathrm{s}$. Therefore, the dense water anomaly and the cyclonic swirl velocity have to be dynamically linked resulting in their simultaneous propagation at this swift speed. A detailed analysis of the dynamics associated with the composite eddy is beyond our current scope. However, we note that the theoretical model of Swaters and Flierl (1991) and Poulin and Swaters (1999) predicts a flow structure similar to our observations and, as such, is a 
good model to explain the dynamics of fully developed DSOW cyclones.

\subsection{Vertical structure of the composite cyclone}

We now investigate the vertical structure of the composite cyclone, although recall that the velocity data above $260 \mathrm{~m}$ and below $660 \mathrm{~m}$ are more sparse, and, as such, the results are not as robust in these two regions.

[Figure 13 about here.]

The background flow $u_{b}+u_{b}^{\prime}(z)$ increases with depth. For simplicity we assume a simple linear fit where constrained by data, and a constant extrapolation elsewhere (Figure 13a). This gives a velocity of $0.36 \mathrm{~m} / \mathrm{s}$ at $900 \mathrm{~m}$ near the bottom (our observations do not extend into the bottom boundary layer). Subtracting this depth-dependent background velocity reveals the vertical structure of the composite eddy (Figure 14). It can again be seen that the peak in the downstream velocity is behind the center, which is due to the velocity in the wake of the cyclone. The cross-stream velocity is roughly symmetric about the eddy center. To further quantify the vertical structure of the cross-stream velocity, a Gaussian eddy was fit to the azimuthal velocity component for each $10 \mathrm{~m}$ depth bin in the same way that it was done for the depth-mean structure (see Figure 11a). While there was little variation (less than $0.5 \mathrm{~km}$ ) in the fitted radius $R_{0}$ from top to bottom, there was a substantial difference in the amplitude $v_{0}$. Therefore, the same calculation was repeated, but with the radius fixed to the depth-mean value of $R_{0}=7.8 \mathrm{~km}$. The resulting amplitudes of the azimuthal velocity are shown in Figure 13b. The vertical structure is well-represented by a quadratic fit with zero velocity at the bottom and zero vertical shear at the top. This implies that the DSOW cyclones at this location on the slope are surface-intensified, with a maximum azimuthal velocity of $0.34 \mathrm{~m} / \mathrm{s}$ near the surface.

[Figure 14 about here.] 
[Figure 15 about here.]

As noted earlier, the moored profilers measuring the hydrographic properties were not able to complete full-depth profiles in the presence of the large velocities inside of the DSOW cyclones. However, on the offshore side of the cyclones, the upstream directed azimuthal velocity leads to a relatively weak total velocity (Figure 8) and the profilers did perform comparatively well there. This allows us to use data from mooring EG5 to construct a composite of the density field approximately $7-10 \mathrm{~km}$ seaward of the cyclone center (Figure 15). Along this slice the density strongly increases near the bottom. From a Eulerian point of view, one sees that the waters denser than $\approx 27.7 \mathrm{~kg} / \mathrm{m}^{3}$ are raised by more than $200 \mathrm{~m}$ during the passage of the cyclones compared to the ambient conditions. In contrast, the depths of the overlying isopycnals are only weakly affected, and this leads to a significant increase of the stratification in the middle of the water column (around $600 \mathrm{~m}$ depth). The downstream velocity in the lower part of the water column where the density anomaly is large is faster than in the upper part (Figure 14a). This means that the dense fluid is advected faster than the overlying water with the ambient density structure. Figures 14 and 15 demonstrate that the density anomaly associated with DSOW cyclones is swiftly advected in the lower part of the water column while the azimuthal velocity is strongest in the upper part of the water column. This is very much in line with the laboratory and numerical results of Whitehead et al. (1990) and the theory of Swaters and Flierl (1991) wherein a propagating lens of dense water near the bottom is associated with an overlying cyclonic vortex.

\subsection{Constructed pressure and density fields of the composite cyclone}

The density field of a DSOW cyclone is of interest both to determine its equatorward transport of dense water as well as to infer its sea surface signature. As such, we now apply an indirect method, using the pressure field, to estimate the density field. The EG4 data used are the ADCP measurements of velocity, microcat measurements of bottom density, 
and, outside of the cyclones, the moored profiler measurements of density (which are limited to $150-650 \mathrm{~m})$. To reduce the noise we apply fits to each of these quantities. The resulting mean background density profile is shown in Figure 13c. We applied two piecewise linear fits to the profile (the top $200 \mathrm{~m}$, where the fit is poor, has little bearing on the result below).

[Figure 16 about here.]

To construct the pressure field we first simplify the radial momentum equation in the frame of reference moving with the cyclone by neglecting friction, time-dependence, and any non-linear terms not associated with the cyclostrophic balance. This also neglects any asymmetries between the onshore and offshore sides of the eddy introduced by the moving frame of reference used here. To leading order the following analysis holds, but we note that the neglected terms may result in quantitative differences. The dynamic pressure can be determined from the integral of the geostrophic and the cyclostrophic terms in the simplified radial momentum equation

$$
p^{\prime}(r)=\rho_{0} \int_{-\infty}^{r}\left(f v\left(r^{\prime}\right)+\frac{v^{2}\left(r^{\prime}\right)}{r^{\prime}}\right) d r^{\prime}
$$

with the boundary condition that the pressure anomaly vanishes far outside of the cyclone. This boundary condition is equivalent to the "Stern integral constraint" (Mory, 1985) and allows the cyclone to be balanced independently of far-field interactions. Hence we need to know the azimuthal velocity as a function of radius and depth, which is obtained from the fits in Figures 11a and 13b, and shown in Figure 16a. We then perform the integration in Equation (6) at each depth to obtain the dynamic pressure field which is shown in Figure 16b. A maximum dynamic pressure anomaly of $-700 \mathrm{~Pa}$ is achieved at the surface in the center of the cyclone. This corresponds to a sea surface height depression of about $7 \mathrm{~cm}$, which compares well with the median SSH depression of $6 \mathrm{~cm}$ in the numerical model of Käse et al. (2003). 
Next we use the hydrostatic equation to obtain the density anomaly:

$$
\rho^{\prime}(z)=-\frac{1}{g} \frac{\partial p^{\prime}}{\partial z}
$$

611

This density anomaly field is shown in Figure 16c. Its radial structure at the bottom is shown in Figure 16d compared to that measured by the microcat. The two curves have the same Gaussian structure with a radius of $7.8 \mathrm{~km}$. The only difference is that the amplitude of the density anomaly computed from the dynamic pressure is roughly 1.8 times larger than that measured by the microcat. We note, however, that both of these estimation methods are uncertain $(\approx 20 \%$ each). Additionally, Equation $(6)$ neglects several terms in the momentum balance. At 13-15 m above the bottom, the microcat may also be located in the $O(10-100 \mathrm{~m})$ thick bottom boundary layer where enhanced mixing could lead to a weaker $\frac{\partial \rho}{\partial r}$ than in the fluid above. Additionally, we do not take into consideration the large (factor of 2-3) change in total water depth between the onshore side and the offshore side of an eddy. In light of these considerations, we suspect that the true bottom density anomaly lies somewhere between the two estimates in Figure 16d. Therefore, we take the two estimates as upper and lower bounds and present the respective full density fields.

If we add the density anomaly field obtained from the pressure gradient calculation to the full density profile outside of the cyclones (Figure 13c), we obtain the full density field shown in Figure 16e. If we divide the density anomaly field (Figure 16c) by 1.8 (the ratio between the two anomalies in Figure 16d) and add that to the density profile outside of the cyclones, we obtain the full density field in Figure 16f. Note the good qualitative agreement between these two inferred density fields and the (independent) measurements on the seaward side of the cyclones (Figure 15). From Figures 16e,f we conclude that the 27.8 isopycnal extends 60-300 $\mathrm{m}$ above the bottom and is confined inside a radius of $4-10 \mathrm{~km}$ around the cyclone center. This again compares well with the $250 \mathrm{~m}$ median plume thickness found by Käse 
et al. (2003). The total volume of water denser than 27.8 in a typical DSOW cyclone at the $900 \mathrm{~m}$ isobath is thus estimated to be $2-45 \mathrm{~km}^{3}$.

We note that the center of the composite cyclone is at a water depth where the densest ambient water is typically 27.74 , which is more than $0.06 \mathrm{~kg} / \mathrm{m}^{3}$ lighter than the traditional DSOW definition of 27.8. Therefore, it might be more appropriate to consider the water coming from the overflow as that comprising the density classes which are otherwise absent at this depth. The 27.74 isopycnal is inside of a radius of about $20 \mathrm{~km}$ and rises about 300-370 m above the bottom (Figures 16e,f). Therefore a typical DSOW cyclone contains $130-200 \mathrm{~km}^{3}$ of water denser than 27.74 .

If we divide the volume of overflow water inside a typical DSOW cyclone by the period over which the cyclones pass the mooring array (2 days), we obtain the volume transport of plume water due to the cyclones. Using the typical definition of overflow water (27.8), we obtain a transport of $0.01-0.26 \mathrm{~Sv}$. Using the broader definition (27.74), we obtain a transport of $0.7-1.2 \mathrm{~Sv}$. Compared to a total overflow water (>27.8) transport of 5.2 $\mathrm{Sv}$ in this region (Dickson and Brown, 1994), these values are rather small. However, this is due to the fact that the cyclones are so high up on the slope (at EG4 in $900 \mathrm{~m}$ ) and hence do not contain much DSOW. Since the background density at greater depth is already larger than the overflow water density criterion, cyclones in deeper water contain disproportionately more overflow water than cyclones around $900 \mathrm{~m}$ depth. Even though their number is small (Figure 6), the overflow transport estimate would increase significantly if we could include the exact dimensions of these deeper eddies in our estimates.

\section{Investigating the Sea Surface Temperature Signature of DSOW Cyclones}

The sea surface temperature along the East Greenland slope is dominated by the contrast between cold $\left(\approx 0-4^{\circ} \mathrm{C}\right)$ polar-origin water on the shelf and warm $\left(\approx 8-12^{\circ} \mathrm{C}\right)$ subtropicalorigin water in the Irminger basin. This water mass front is associated with the surface- 
intensified East Greenland/Irminger Current. The $6^{\circ} \mathrm{C}$ SST isotherm is a good proxy for the frontal location. Using the 63 partially cloud-free SST images obtained between September 2007 and October 2008, we produced a map of the frontal locations in the vicinity of the mooring array (Figure 17). It can be seen that the front meanders substantially. Its mean location roughly tracks the $500 \mathrm{~m}$ isobath (slightly deeper than the shelfbreak), and the standard deviation is about $10 \mathrm{~km}$. The mooring array brackets the frontal location to within \pm 1 standard deviation. The median location of the DSOW cyclones passing by the mooring array is a few kilometers offshore of the mean location of the $6^{\circ} \mathrm{C}$ isotherm.

[Figure 17 about here.]

The approximate co-location of the SST front and the path of DSOW cyclones means that the surface velocity field of the cyclones is generally in close vicinity to the SST front and will likely impact the front. Disturbances in SST and anomalies in DSOW transport associated with DSOW cyclones were seen to move along the East Greenland slope together for six days in the numerical model of Magaldi et al. (2011). The premise that SST disturbances and DSOW cyclones move together was also used by Bruce (1995) to track cyclones. The 33 SST disturbances identified by Bruce (1995) along the $\approx 700 \mathrm{~km}$ of the East Greenland shelfbreak were found to progress equatorward at $0.27 \mathrm{~m} / \mathrm{s}$ with a standard deviation of $0.11 \mathrm{~m} / \mathrm{s}$. This was subsequently interpreted as the typical propagation velocity of DSOW cyclones along the East Greenland slope. As shown above (Subsection 5.2), our in-situ measurements suggest a typical translational velocity with respect to the bottom of $0.72 \mathrm{~m} / \mathrm{s}$. We now examine possible reasons for this large discrepancy.

[Figure 18 about here.]

One simple hypothesis would be that there is significant interannual variability in DSOW cyclone properties and that during 2007-08 they translated faster than during 1987-90 (the 
time period analyzed by Bruce (1995)). In order to address this hypothesis, we repeated the methodology of Bruce (1995) for all SST images between September 2007 and October 2008 that were at least partially cloud-free. Along the first $250 \mathrm{~km}$ of the East Greenland shelfbreak, this revealed several spiral or hook-like features on the SST front qualitatively and quantitatively similar to the ones shown in Figure 2 of Bruce (1995). Over this yearlong period, 58 SST disturbances could be identified in more than one SST image, and their tracks are shown in Figure 18. Note that the number of SST disturbances at the mooring array location is very small (roughly five) and a statistically meaningful comparison between individual features in SST and the mooring record is thus not possible. Of the 58 disturbances, 40 were trackable in the sense that they were identified in SST images more than 6 hours apart. Their propagation speed was $0.37 \mathrm{~m} / \mathrm{s}$ with a standard deviation of $0.17 \mathrm{~m} / \mathrm{s}$. This is slightly faster than the mean of Bruce (1995), but the two estimates agree within their standard deviations. Hence interannual variability cannot by itself explain the observed difference.

Both the topographic Rossby wave speed and the Nof speed are proportional to the bottom slope. As such, a second hypothesis to explain the discrepancy between our results and those of Bruce (1995) is that the bottom slope at the mooring array is steeper than elsewhere, thereby accounting for the faster propagation speeds determined from the mooring array data. We analyzed the General Bathymetric Chart of the Oceans (GEBCO) at 30 arc second resolution to determine the bottom slope near the $900 \mathrm{~m}$ isobath. West of $35^{\circ} \mathrm{W}$ the slope becomes much steeper (Figure 1). However, the bottom slope within $100 \mathrm{~km}$ of the mooring array where we tracked the SST disturbances (Figure 18) varies by only about $\pm 50 \%$ of the value at the array site. Based on this simple argument, one might expect that, compared with the value at the mooring array location, the propagation speed along the East Greenland slope is both faster in some places and slower in other places with a mean not greatly different from the value at the mooring array location. The varying bottom slope 
can therefore also not fully explain the difference in the observed speeds.

We propose a third hypothesis to explain the difference between the DSOW cyclone insitu translational velocity and the propagation speed of the SST disturbances; in particular, that the SST disturbances are not propagating with the DSOW cyclones. In this scenario the SST disturbances could be initially generated by some process (e.g. DSOW cyclones just downstream of the sill or baroclinic instability of the EGC/IC front) and then propagate independently of the deep DSOW cyclones along the East Greenland slope. Lozier et al. (2002) studied the propagation speed associated with meanders of the Mid-Atlantic Bight shelfbreak front. They found that the meanders propagate at a velocity slower than or equal to the mean surface speed of the frontal jet. Their situation is similar to the EGC/IC front suggesting that once the SST disturbances are generated, their speed will not exceed the mean surface velocities. Using the mooring data from the present array, in the absence of cyclones, von Appen (2012) found the near-surface EGC/IC frontal jet speed to be $\approx 0.25 \mathrm{~m} / \mathrm{s}$, which is in line with the speed of the SST disturbances calculated above and by Bruce (1995). In the model of Swaters and Flierl (1991) and in our mooring observations (Section 5), the azimuthal velocity field of the cyclone cannot kinematically trap fluid and only the dense water in the lower part of the eddy moves at the fast propagation speed. Nonetheless, there may be times during the evolution of DSOW cyclones when the peak azimuthal velocity $v_{0}(z=0)$ is greater than the propagation speed $u_{t}$. For $u_{t} / v_{0}<1$ kinematic trapping would occur (Flierl, 1981). For the duration of such kinematic trapping, the cyclones could form spiral and hook like features in the SST field. As the cyclones evolve, the ratio $u_{t} / v_{0}$ might decrease to values below one (at the mooring array location it is $\approx 0.76$ ). At this point the cyclones would no longer trap the fluid near the sea surface leaving the SST disturbances they generated behind to propagate with the mean surface speed. While this scenario is consistent with previous studies on DSOW cyclones and the observations presented here, future investigations are required to test its validity. 


\section{Summary}

Using data from a cross-shelfbreak mooring array $280 \mathrm{~km}$ downstream of Denmark Strait, we identified 190 DSOW cyclones. On average, an eddy passed the array every other day, most of them near the $900 \mathrm{~m}$ isobath. The composite velocity field of a DSOW cyclone in the middle water column $(260-660 \mathrm{~m})$ shows that the features typically propagate at $0.47 \mathrm{~m} / \mathrm{s}$ with respect to the mean flow, which is consistent with the propagation of a topographic Rossby wave and the Nof speed. Their propagation velocity with respect to the bottom is $0.72 \mathrm{~m} / \mathrm{s}$, and they have a peak depth-mean azimuthal velocity of $0.22 \mathrm{~m} / \mathrm{s}$ at a radius of $7.8 \mathrm{~km}$. These values are substantially different from the statistics presented by Bruce (1995) and the 25-35 km distance between consecutive eddies seen in the numerical model of Spall and Price (1998). We propose a scenario in which the SST disturbances tracked by Bruce (1995) would propagate with the mean flow of the East Greenland/Irminger Current rather than with the DSOW cyclones underneath. This could be an explanation for the differences between the results of Bruce (1995) and our study as contrasted in Table 1.

[Table 1 about here.]

We have shown the DSOW cyclones to be energetic contributors to the variability several hundred kilometers downstream of Denmark Strait. However, such variability is not observed farther south along the East Greenland slope in the vicinity of Cape Farewell (e.g. Bacon and Saunders, 2010; Daniault et al., 2011). This implies that the cyclones decay, but the mechanisms by which this happens and the distance over which this occurs remain to be investigated.

\section{Acknowledgments}

The authors wish to thank Paula Fratantoni, Frank Bahr, and Dan Torres for processing the mooring data. The mooring array was capably deployed by the crew of the $R / V \overline{A r n i}$ 
Friðriksson and recovered by the crew of the $R / V$ Knorr. We thank Héðinn Valdimarsson for his assistance in the field work. We are grateful for helpful discussions with Paola Rizzoli, Mike Spall, and Amy Bower. Three anonymous reviewers provided valuable suggestions. Creation of the MODIS satellite data sets is credited to the NASA Ocean Biology Processing Group (OBPG), the NASA Jet Propulsion Laboratory (JPL), and the University of Miami Rosenstiel School of Marine and Atmospheric Science (RSMAS). Funding for the study was provided by National Science Foundation Grant OCE-0726640 and the Arctic Research Initiative of the Woods Hole Oceanographic Institution.

\section{References}

Bacon, S., Saunders, P., 2010. The Deep Western Boundary Current at Cape Farewell: Results from a Moored Current Meter Array. Journal of Physical Oceanography 40 (4), 815-829.

Brearley, J., Pickart, R., Valdimarsson, H., Jónsson, S., Schmitt, R., Haine, T., 2012. The East Greenland Boundary Current System South of Denmark Strait. Deep Sea Research Part I 63 (1), 1-19.

Brown, O., Minnett, P., 1999. MODIS Infrared Sea Surface Temperature Algorithm: Algorithm Theoretical Basis Document, Version 2.0. Tech. rep., University of Miami, available at http://modis.gsfc.nasa. gov/data/atbd/atbd_mod25.pdf.

Bruce, J., 1995. Eddies Southwest of the Denmark Strait. Deep Sea Research Part I: Oceanographic Research Papers 42 (1), 13-17.

Daniault, N., Lherminier, P., Mercier, H., 2011. Circulation and Transport at the Southeast Tip of Greenland. Journal of Physical Oceanography 41, 437-457.

Dickson, R., Brown, J., 1994. The Production of North Atlantic Deep Water: Sources, Rates, and Pathways. Journal of Geophysical Research 99 (C6), 12319-12341.

Flierl, G., 1981. Particle Motions in Large-Amplitude Wave Fields. Geophysical \& Astrophysical Fluid Dynamics 18 (1-2), 39-74.

Girton, J., Sanford, T., Käse, R., 2001. Synoptic Sections of the Denmark Strait Overflow. Geophysical Research Letters 28 (8), 1619-1622.

Haine, T. W., 2010. High-Frequency Fluctuations in Denmark Strait Transport. Geophysical Research Letters 37 (14), L14601. 
Haine, T. W., Zhang, S., Moore, G. W. K., Renfrew, I. A., 2009. On the Impact of High-Resolution, HighFrequency Meteorological Forcing on Denmark-Strait Ocean Circulation. Quarterly Journal of the Royal Meterological Society.

Harden, B., Renfrew, I., Petersen, G., 2011. A Climatology of Wintertime Barrier Winds off Southeast Greenland. Journal of Climate 24, 4701-4717.

Harden, B. E., Pickart, R. S., Renfrew, I. A., 2013. Offshore Transport of Dense Water from the East Greenland Shelf. Journal of Physical Oceanography accepted.

Jochumsen, K., Quadfasel, D., Valdimarsson, H., Jónsson, S., 2012. Variability of the Denmark Strait Overflow: Moored Time Series from 1996-2011. Journal of Geophysical Research 117 (C12).

Käse, R., Girton, J., Sanford, T., 2003. Structure and Variability of the Denmark Strait Overflow: Model and Observations. Journal of Geophysical Research 108 (C6), 3181.

Krauss, W., 1996. A Note on Overflow Eddies. Deep Sea Research Part I: Oceanographic Research Papers 43 (10), 1661-1667.

Lilly, J., Rhines, P., 2002. Coherent Eddies in the Labrador Sea Observed from a Mooring. Journal of Physical Oceanography $32(2), 585-598$.

Lozier, M., Reed, M., Gawarkiewicz, G., 2002. Instability of a Shelfbreak Front. Journal of Physical Oceanography $32(3), 924-944$.

Macrander, A., Käse, R., Send, U., Valdimarsson, H., Jónsson, S., 2007. Spatial and Temporal Structure of the Denmark Strait Overflow Revealed by Acoustic Observations. Ocean Dynamics 57 (2), 75-89.

Macrander, A., Send, U., Valdimarsson, H., Jónsson, S., Käse, R., 2005. Interannual Changes in the Overflow from the Nordic Seas into the Atlantic Ocean through Denmark Strait. Geophysical Research Letters 32 (6), L06606.

Magaldi, M., Haine, T., Pickart, R., 2011. On the Nature and Variability of the East Greenland Spill Jet: A Case Study in Summer 2003. Journal of Physical Oceanography 41 (12), 2307-2327.

Moore, G., Renfrew, I. A., Pickart, R. S., 2013. Multi-decadal Mobility of the North Atlantic Oscillation. Journal of Climate 26, 2453-2466.

Morrison, A., Billings, J., Doherty, K., Toole, J., 2000. The McLane Moored Profiler: A Platform for Physical, Biological, and Chemical Oceanographic Measurements. In: Proceedings of the Oceanology International 2000 Conference. pp. 1-18.

Mory, M., 1985. Integral Constraints on Bottom and Surface Isolated Eddies. Journal of Physical Oceanography 15 (11), 1433-1438. 
National Geospatial-Intelligence Agency, 2004. Handbook of Magnetic Compass Adjustment. Tech. rep., National Geospatial-Intelligence Agency, Bethesda, MD.

Nof, D., 1983. The Translation of Isolated Cold Eddies on a Sloping Bottom. Deep Sea Research Part A. Oceanographic Research Papers 30 (2), 171-182.

Pawlowicz, R., Beardsley, B., Lentz, S., 2002. Classical Tidal Harmonic Analysis Including Error Estimates in MATLAB Using T_TIDE. Computers and Geosciences 28 (8), 929-937.

Pedlosky, J., 2003. Waves in the Ocean and Atmosphere: Introduction to Wave Dynamics. Springer.

Pickart, R., Torres, D., Fratantoni, P., 2005. The East Greenland Spill Jet. Journal of Physical Oceanography 35 (6), 1037-1053.

Poulin, F., Swaters, G., 1999. Sub-inertial Dynamics of Density-driven Flows in a Continuously Stratified Fluid on a Sloping Bottom. II. Isolated Eddies and Radiating Cold Domes. Proceedings of the Royal Society of London 455 (1986), 2305-2329.

Price, J., O’Neil Baringer, M., 1994. Outflows and Deep Water Production by Marginal Seas. Progress in Oceanography 33 (3), 161-200.

Spall, M., Price, J., 1998. Mesoscale Variability in Denmark Strait: The PV Outflow Hypothesis. Journal of Physical Oceanography 28 (8), 1598-1623.

Sutherland, D., Pickart, R., 2008. The East Greenland Coastal Current: Structure, Variability, and Forcing. Progress in Oceanography 78 (1), 58-77.

Swaters, G., Flierl, G., 1991. Dynamics of Ventilated Coherent Cold Eddies on a Sloping Bottom. Journal of Fluid Mechanics 223, 565-587.

von Appen, W., 2012. Moored Observations of Shelfbreak Processes at the Inflow to and Outflow from the Arctic Ocean. Ph.D. thesis, Massachusetts Institute of Technology and Woods Hole Oceanographic Institution, Cambridge/Woods Hole, MA.

Whitehead, J., Stern, M., Flierl, G., Klinger, B., 1990. Experimental Observations of Baroclinic Eddies on a Sloping Bottom. Journal of Geophysical Research 95 (C6), 9585-9610.

Worthington, L., 1969. An Attempt to Measure the Volume Transport of Norwegian Sea Overflow Water through the Denmark Strait. Deep Sea Research Supplement to Volume 16, 421-432. 


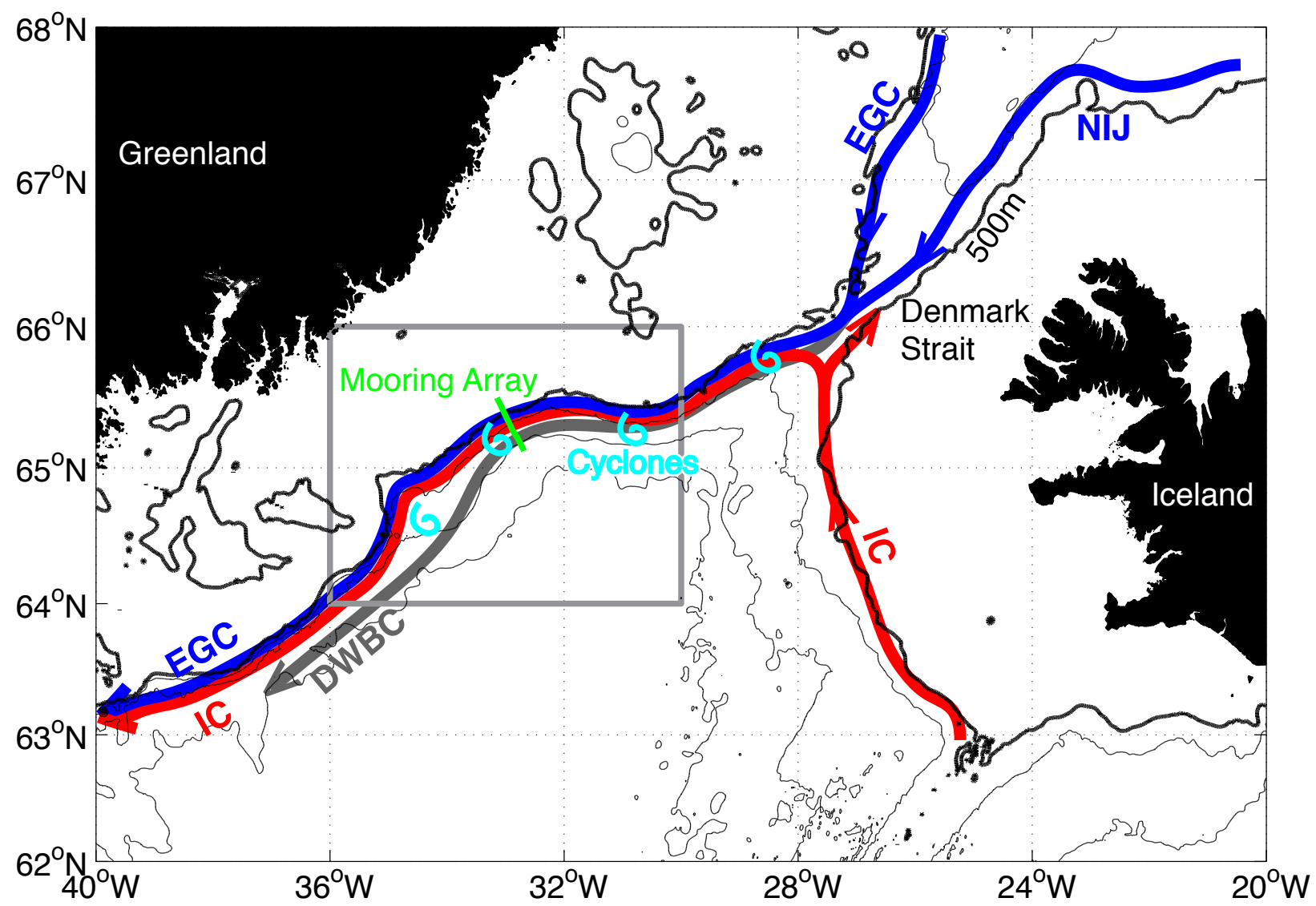

Figure 1: Map of the study region. The location of the mooring array as well as the approximate region populated by DSOW cyclones is shown along with some of the important currents in the region: Irminger Current (IC), East Greenland Current (EGC), North Icelandic Jet (NIJ), and Deep Western Boundary Current (DWBC). South of Denmark Strait, along the East Greenland shelfbreak, the EGC and IC flow together as a single jet referred to in the text as the East Greenland/Irminger Current. 


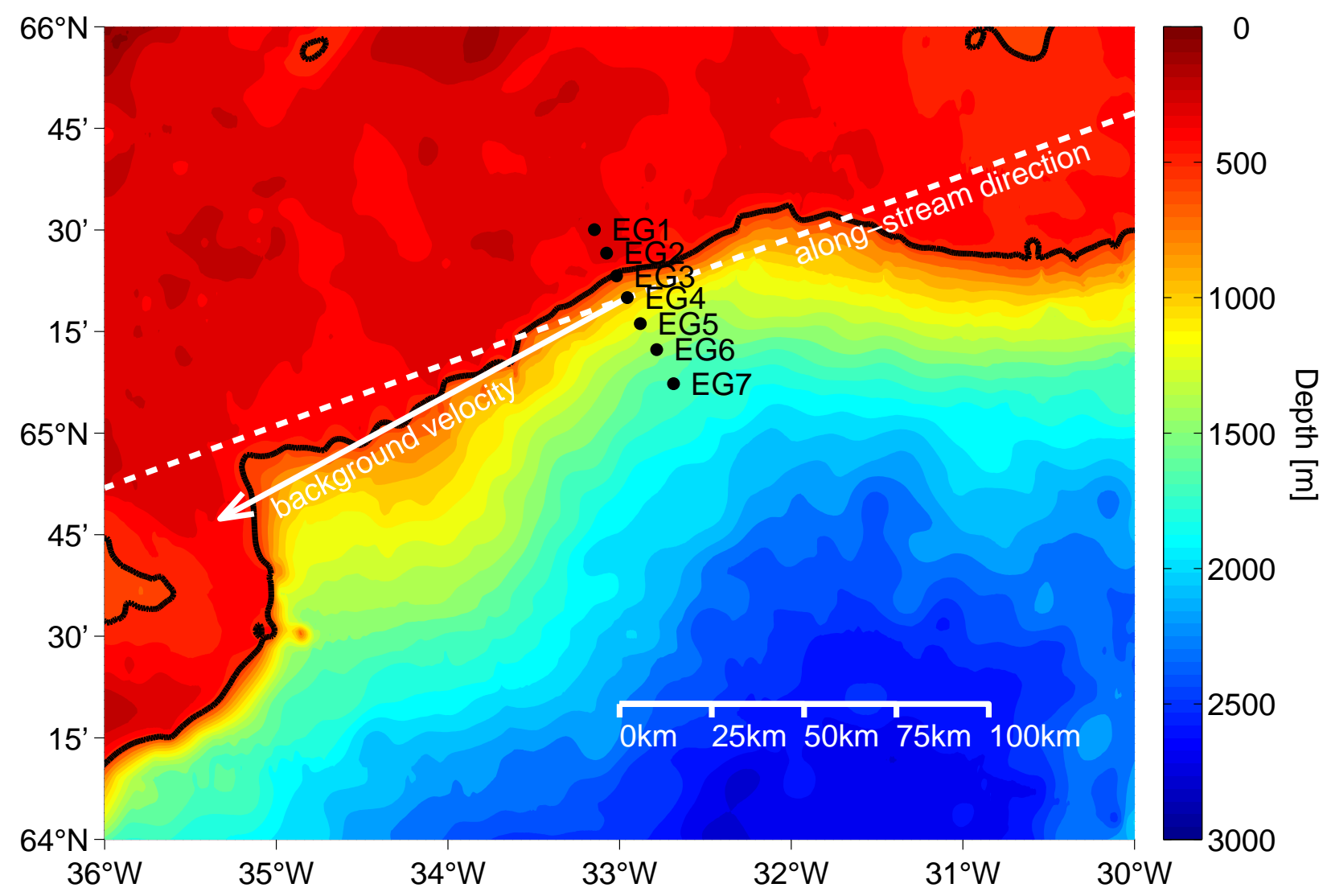

Figure 2: Vicinity of the mooring array with the along-stream direction and the depth-mean background velocity of $0.27 \mathrm{~m} / \mathrm{s}$. Bathymetric data are from the General Bathymetric Chart of the Oceans at 30 arc second resolution. 


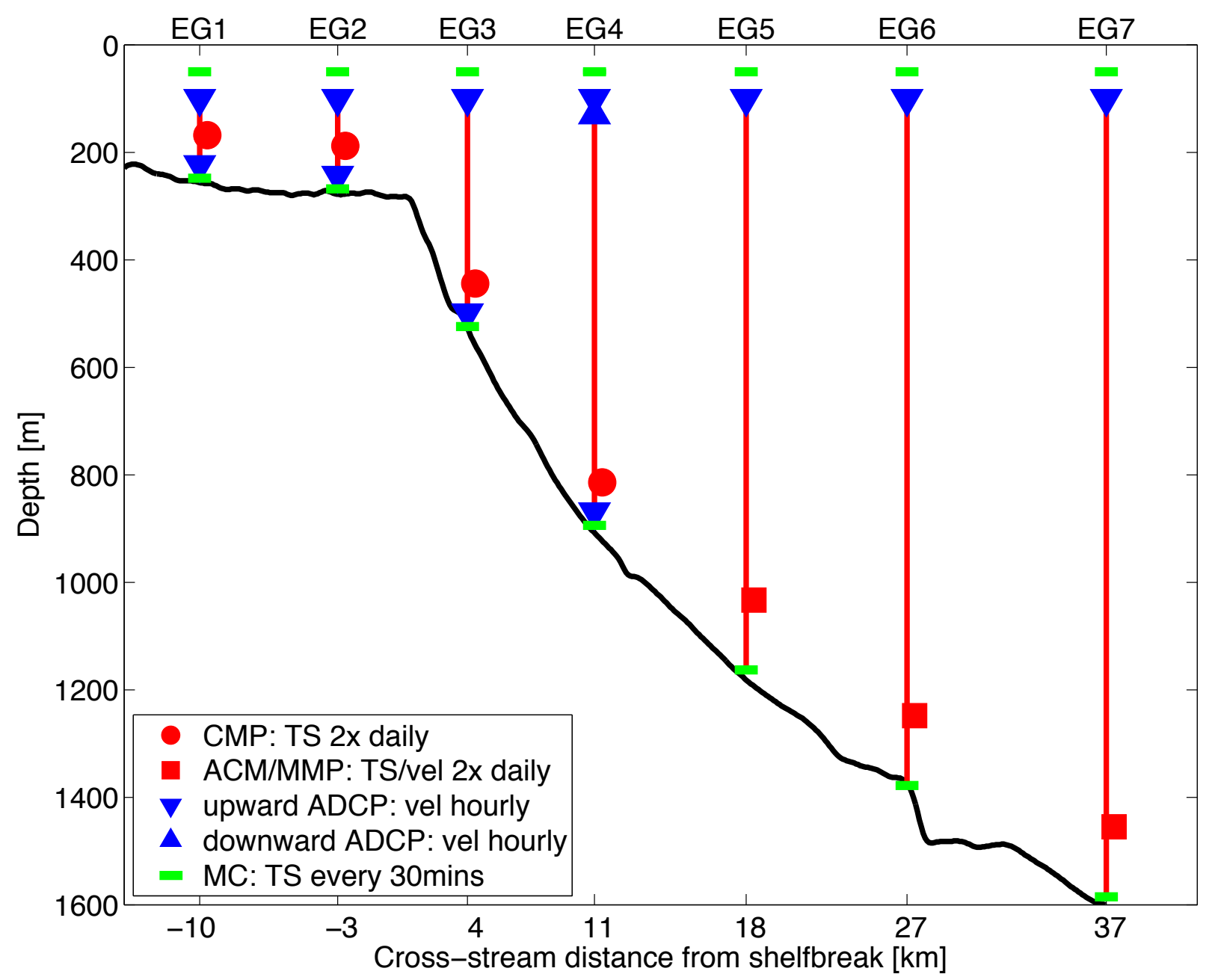

Figure 3: Cross-sectional view of the East Greenland mooring array. The different instruments and their sampling schedules are explained in the legend. The nominal depth range sampled by the CMPs and MMPs is shown in red. The bottom depth along the mooring line, measured by the ship's echo sounder, is shown in black. The acronyms are as follows: CMP: Coastal Moored Profiler, ACM: Acoustic Current Meter, MMP: McLane Moored Profiler, ADCP: Acoustic Doppler Current Profiler, MC: Microcat. 

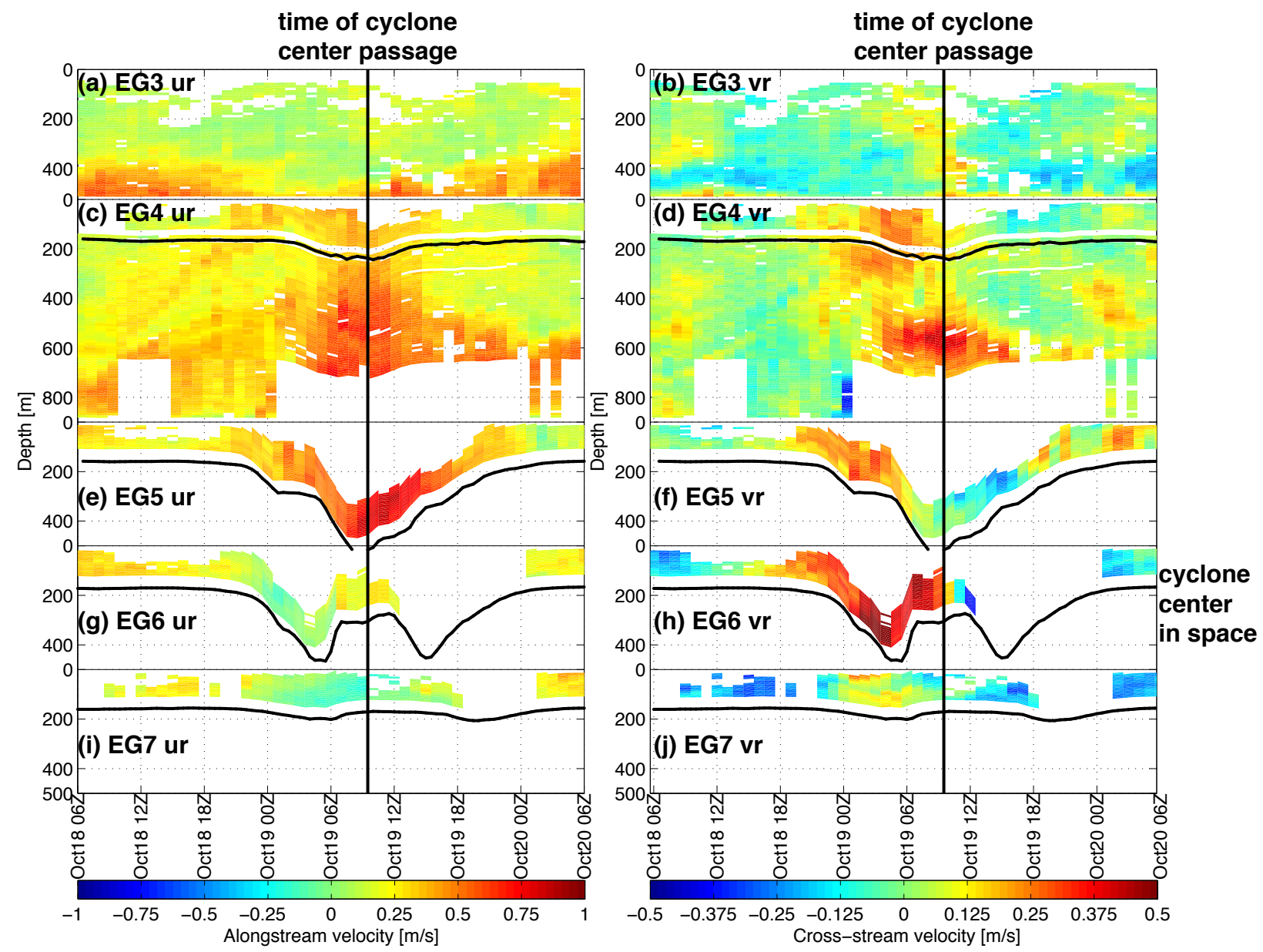

Figure 4: Representative timeseries (from 06Z(06UTC) October 18, 2007 to 06Z October 20, 2007) of velocity (color) and mooring blowdown (black line showing the depth of the top microcat) obtained by the mooring array during the passage of a DSOW cyclone. The left panels show the alongstream velocity, $u$, measured by the ADCPs on the five moorings on the slope. The right panels show the corresponding cross-stream velocity, $v$ (note the difference in velocity scales). The top panels $(\mathrm{a}-\mathrm{d})$ show the full water depth at the moorings (EG3: $525 \mathrm{~m}$, EG4: $900 \mathrm{~m}$ ). Since there were no ADCP velocity records in the lower part of the water column for the outer three moorings, only the measurements in the top $500 \mathrm{~m}$ of the water column are shown in the lower panels (e-j). At 0930Z on October 19, 2007 a cyclone passed onshore of mooring EG6. This time is highlighted with a vertical black line. 


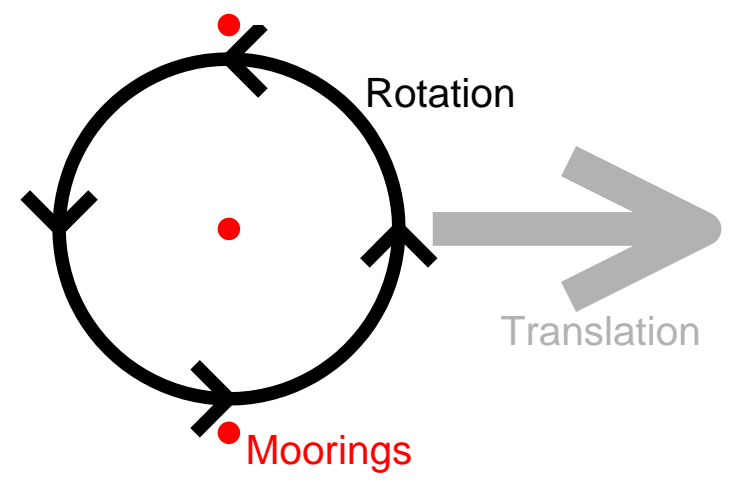

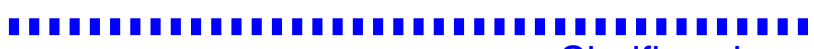

Shelfbreak

Figure 5: Schematic of the flow field when a cyclone translates along the continental slope. The black circle indicates the radius of maximum azimuthal flow. 
(a)

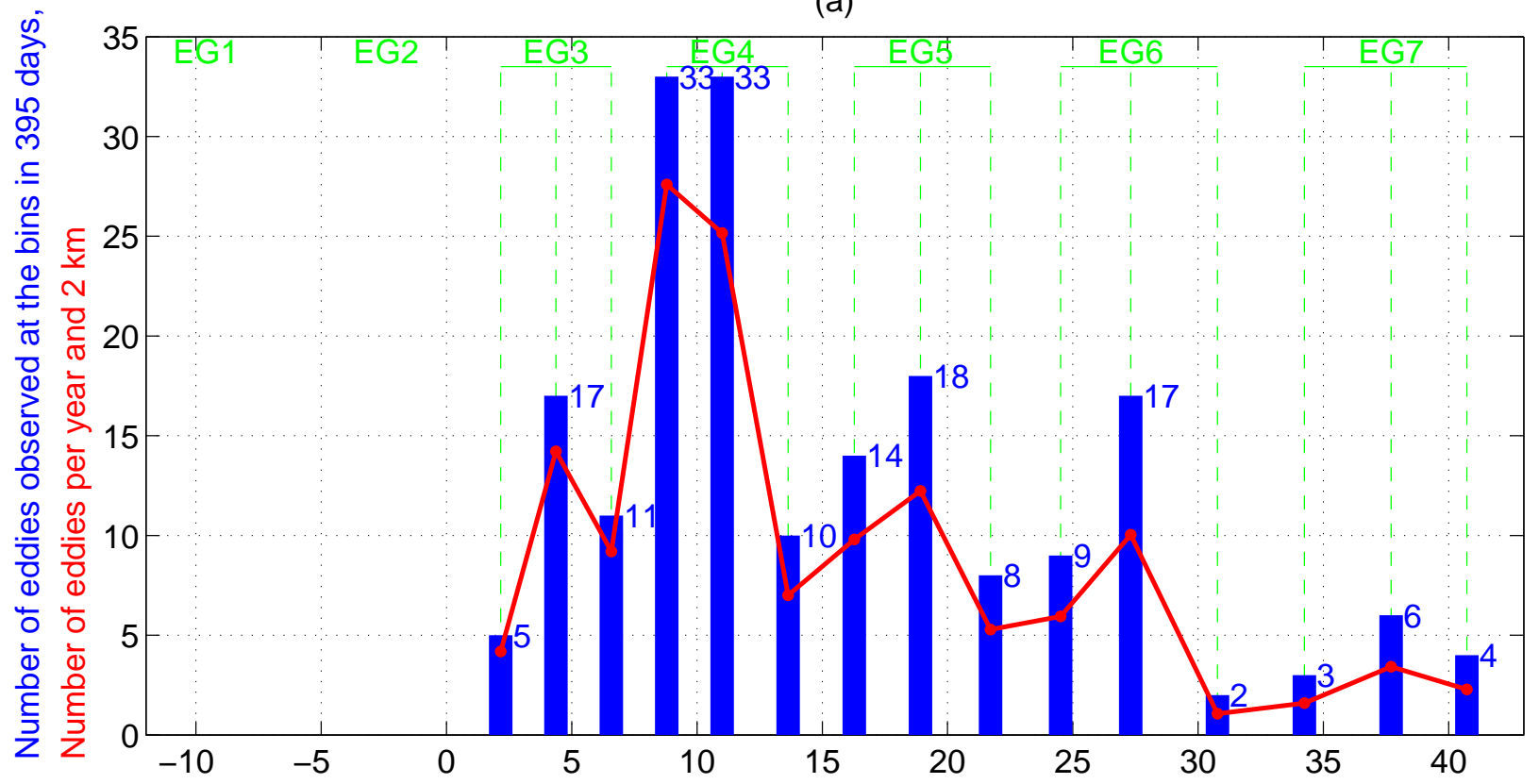

(b)

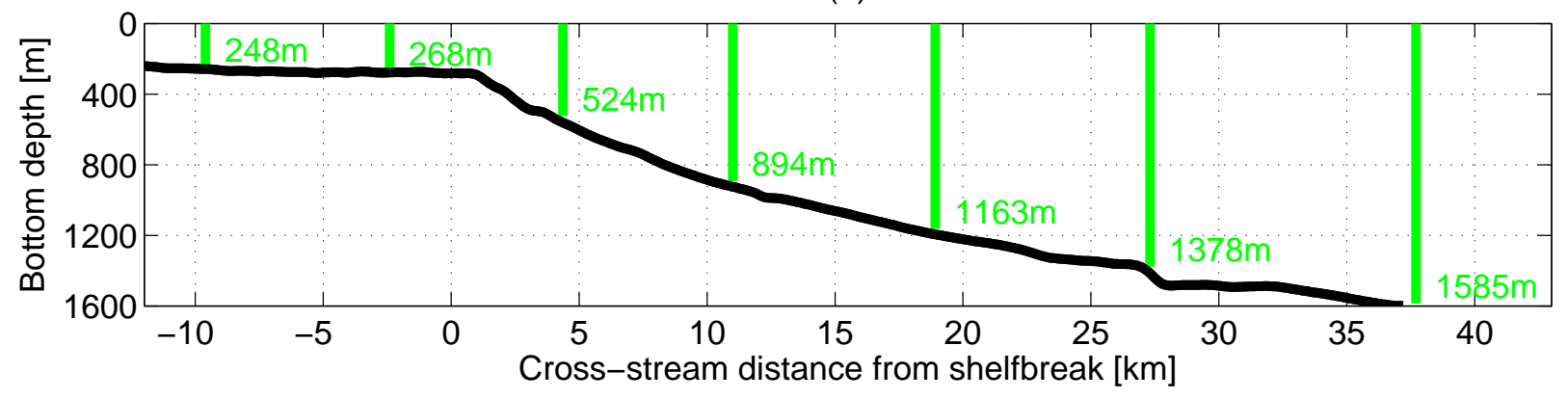

Figure 6: (a) Histogram of cross-stream distribution of the 190 identified eddies (blue bars) over the 395 days of data. The three bins (onshore, near, and offshore) that are assigned to each mooring are shown by the dashed green lines. (b) Cross-stream bottom depth profile. Mooring locations are indicated. 
(a)
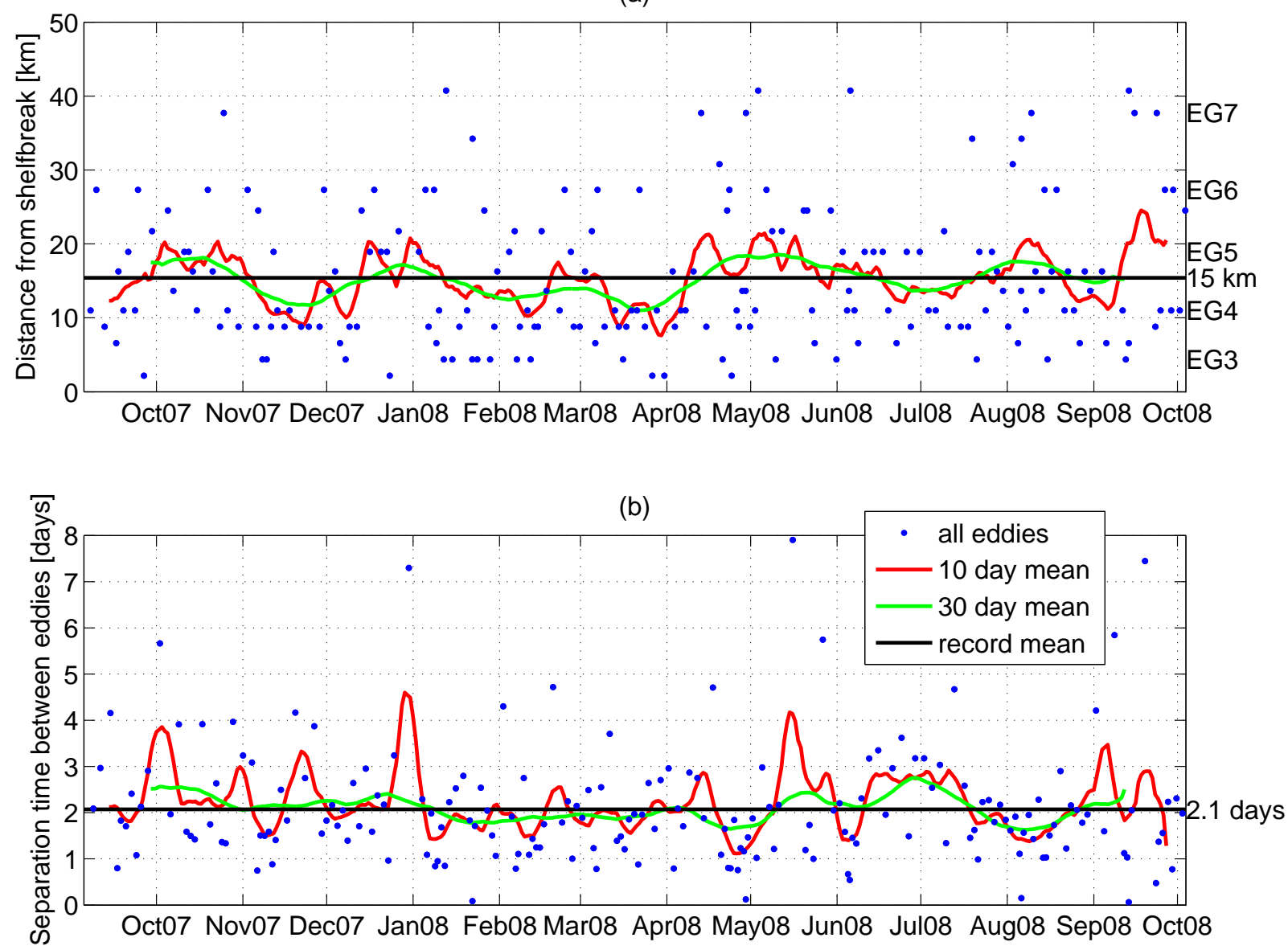

Figure 7: Timeseries of the 190 identified eddies. (a) Cross-stream location at which the eddies (blue dots) were found. Running means as well as the record mean are shown. (b) Time between successive eddies passing the array. 
(a) $(u, v)$ : Depth-mean $(260 m-660 m)$ velocity $[\mathrm{m} / \mathrm{s}]$

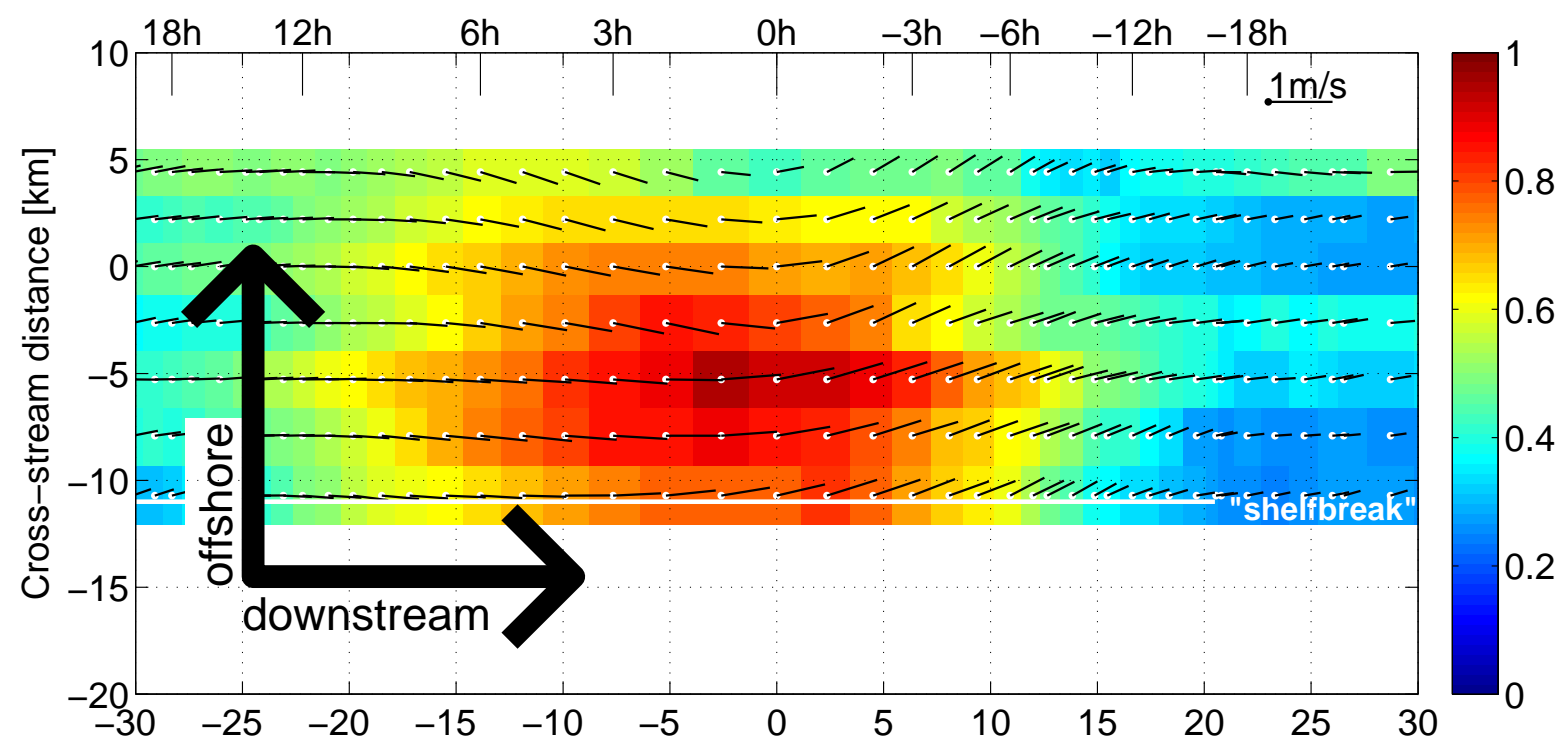

(b)

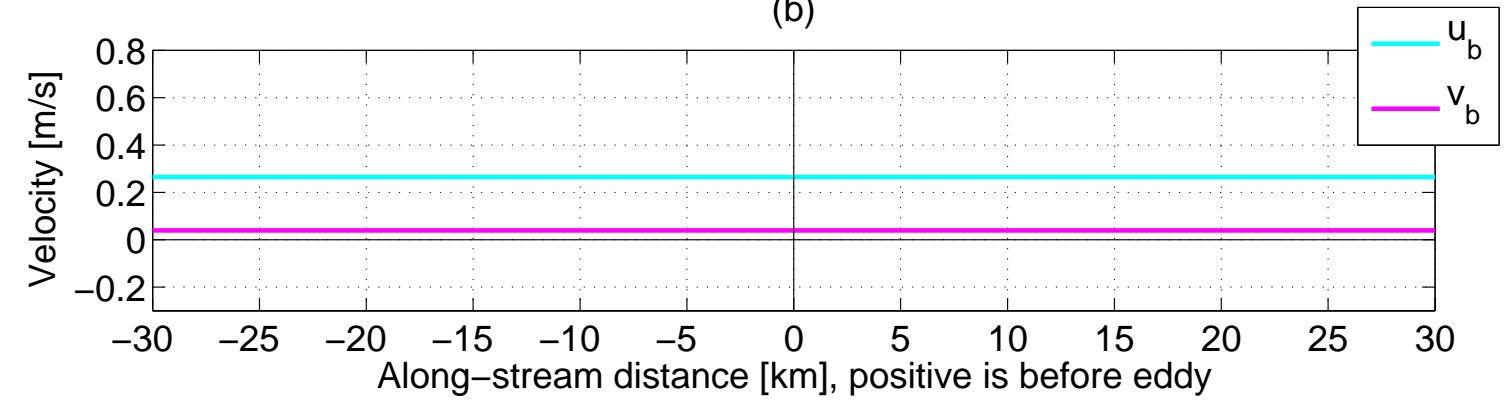

Figure 8: (a) Top-down view of the full depth-mean flow field $(u, v)$ with the center of the cyclone at $(0,0)$. The color of the bins is the speed and the black lines show the vector velocity with a $1 \mathrm{~m} / \mathrm{s}$ scale bar in the top right corner. (b) Background alongstream $u_{b}$ and cross-stream $v_{b}$ velocities in the absence of DSOW cyclones. 
(a) $\left(u-u_{b}, v-v_{b}\right)$ : Depth-mean $(260 m-660 m)$ velocity $[\mathrm{m} / \mathrm{s}]$

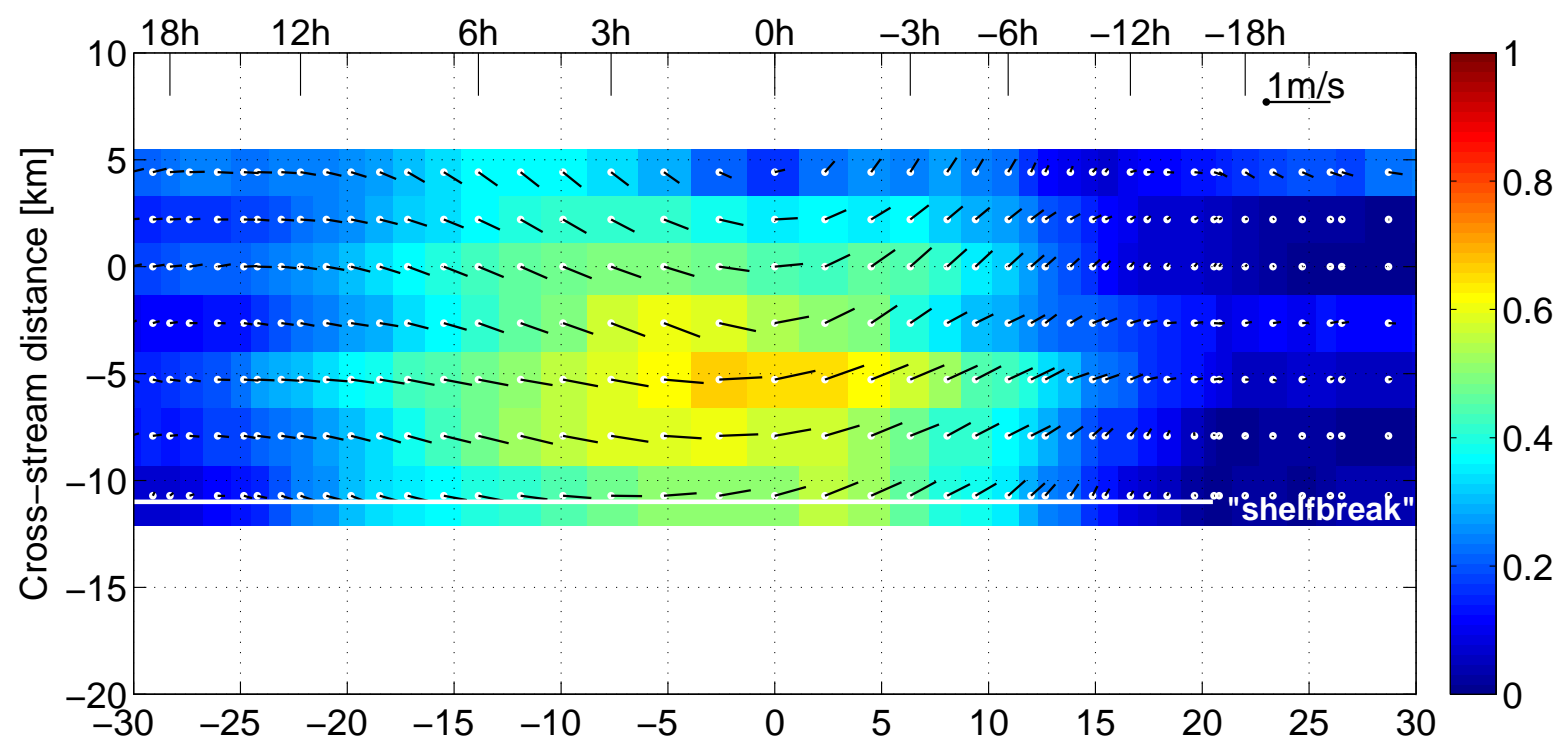

(b)

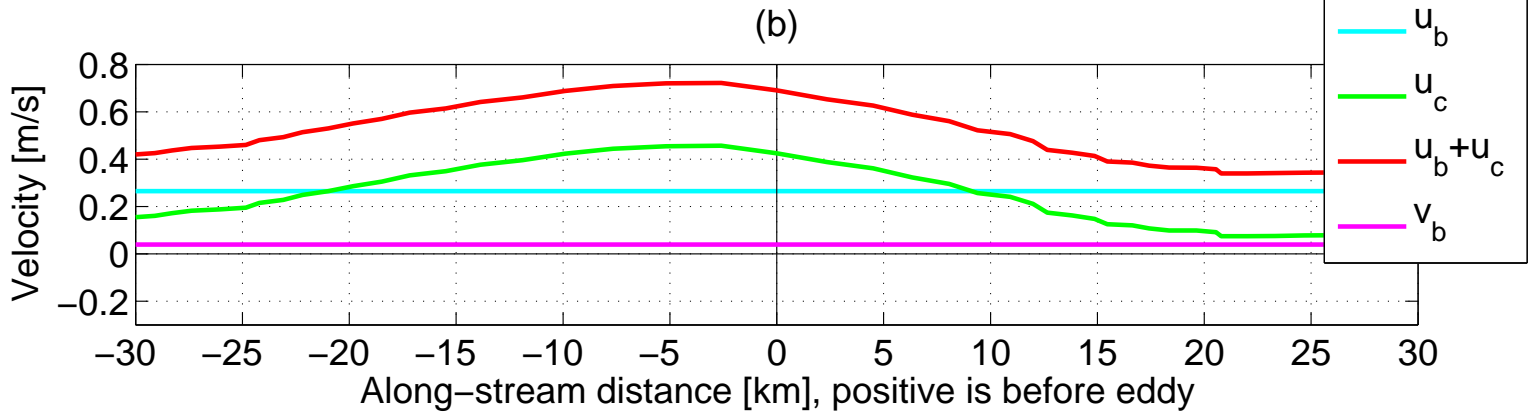

Figure 9: (a) Eddy associated depth-mean flow $\left(u-u_{b}, v-v_{b}\right)$. (b) Co-translational velocity $u_{c}(x)$ of the cyclone with respect to the background mean flow and co-translational velocity $u_{b}+u_{c}(x)$ with respect to the bottom. 
(a) $\left(u-u_{b}-u_{c}, v-v_{b}\right)$ : Depth-mean $(260 m-660 m)$ velocity [m/s]

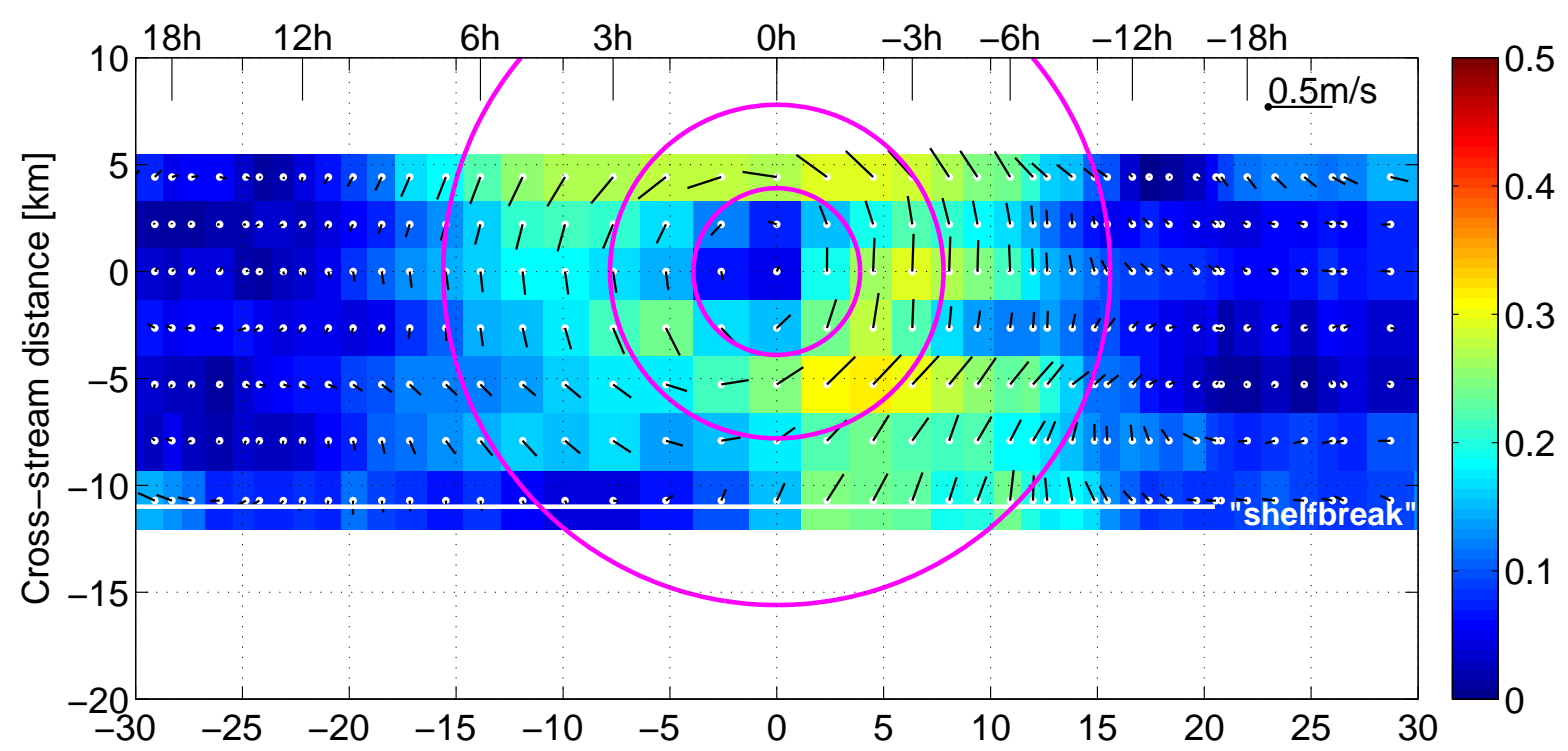

(b)

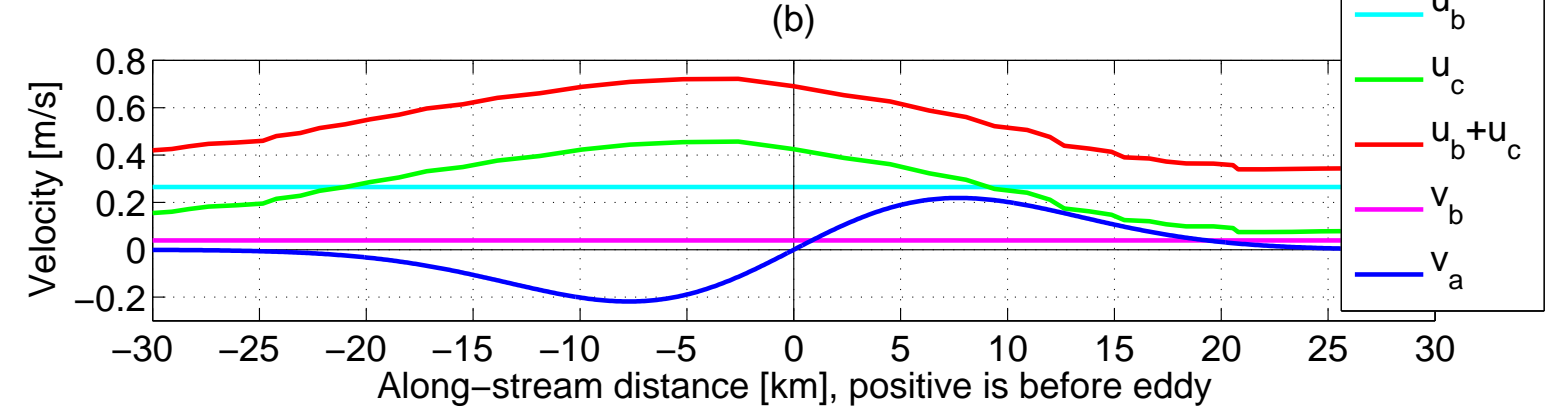

Figure 10: (a) Azimuthal depth-mean flow $\left(u-u_{b}-u_{c}, v-v_{b}\right)$ of the cyclone. Note that the color scale and vector velocity scale have been reduced compared to the previous two figures. Concentric circles are included for visual guidance (radii of $0.5 R_{0}, R_{0}, 2 R_{0}$ where $R_{0}=7.8 \mathrm{~km}$ ). (b) Azimuthal velocity $v_{a}$ of the Gaussian eddy fit from Figure 11a. 
(a) Depth-mean azimuthal velocity

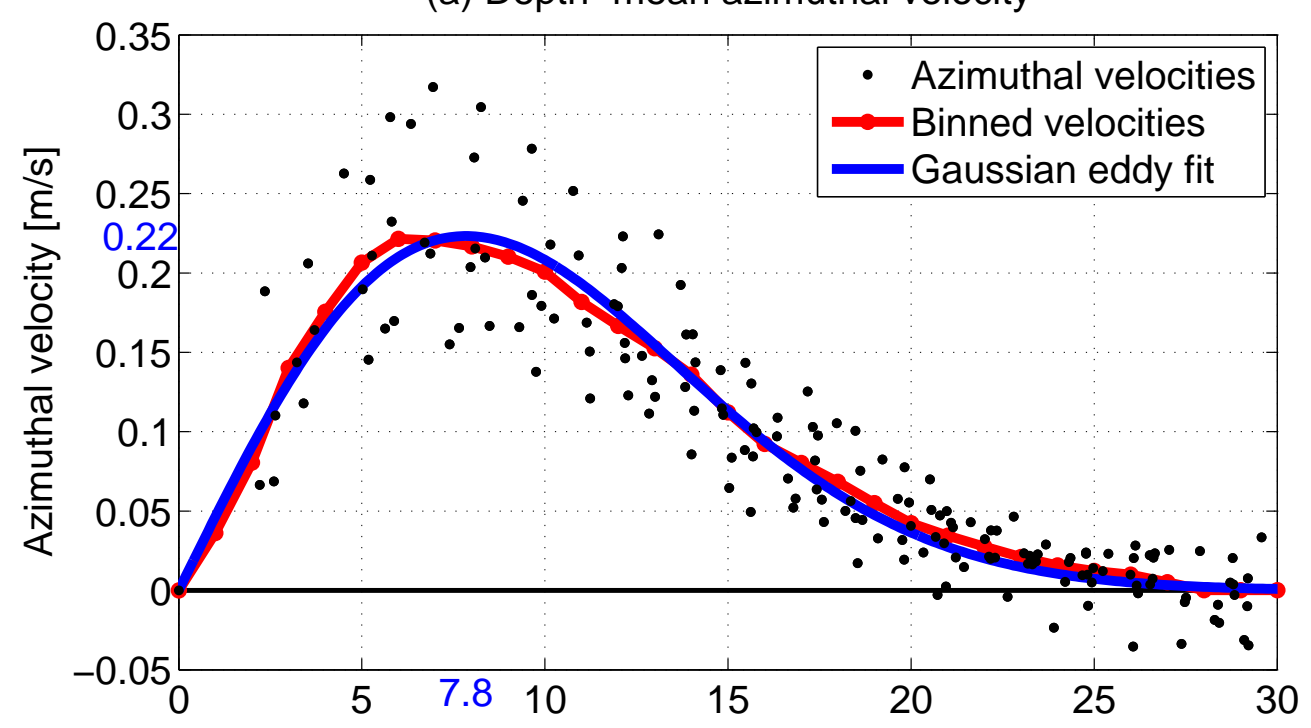

(b) Bottom density anomaly

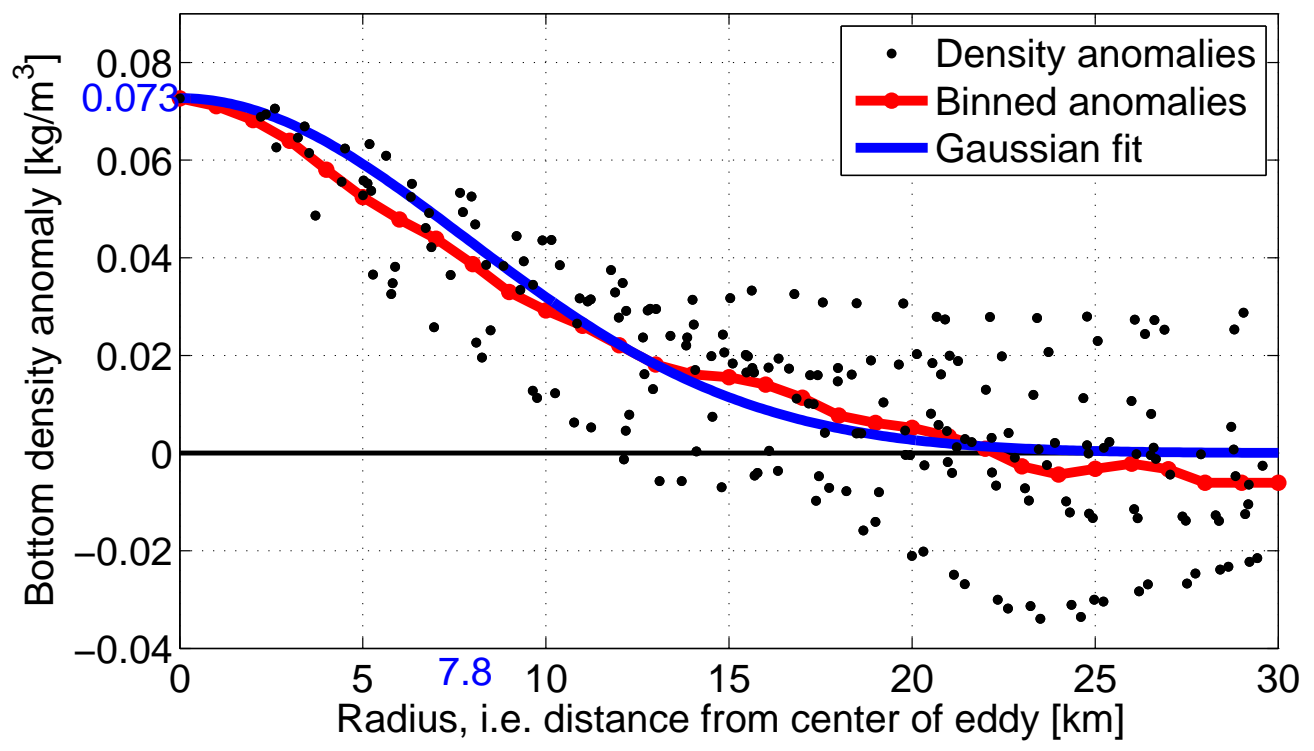

Figure 11: (a) Radial dependance of the azimuthal velocities $v_{a}$ of the composite eddy. The black dots correspond to bins in Figure 10, the red curve is a smoothed $1 \mathrm{~km}$ running mean, and the blue curve is a least squares fit of the Gaussian eddy model to the data. (b) Radial dependence of the bottom density anomaly $\sigma_{e}^{\prime}$ from Figure 12 . 
(a) $\left(\mathrm{u}-\mathrm{u}_{\mathrm{b}}-\mathrm{u}_{\mathrm{c}}, \mathrm{v}-\mathrm{v}_{\mathrm{b}}\right)$ : Depth-mean velocity $[\mathrm{m} / \mathrm{s}]$ and Bottom density anomaly $\left[\mathrm{kg} / \mathrm{m}^{3}\right]$
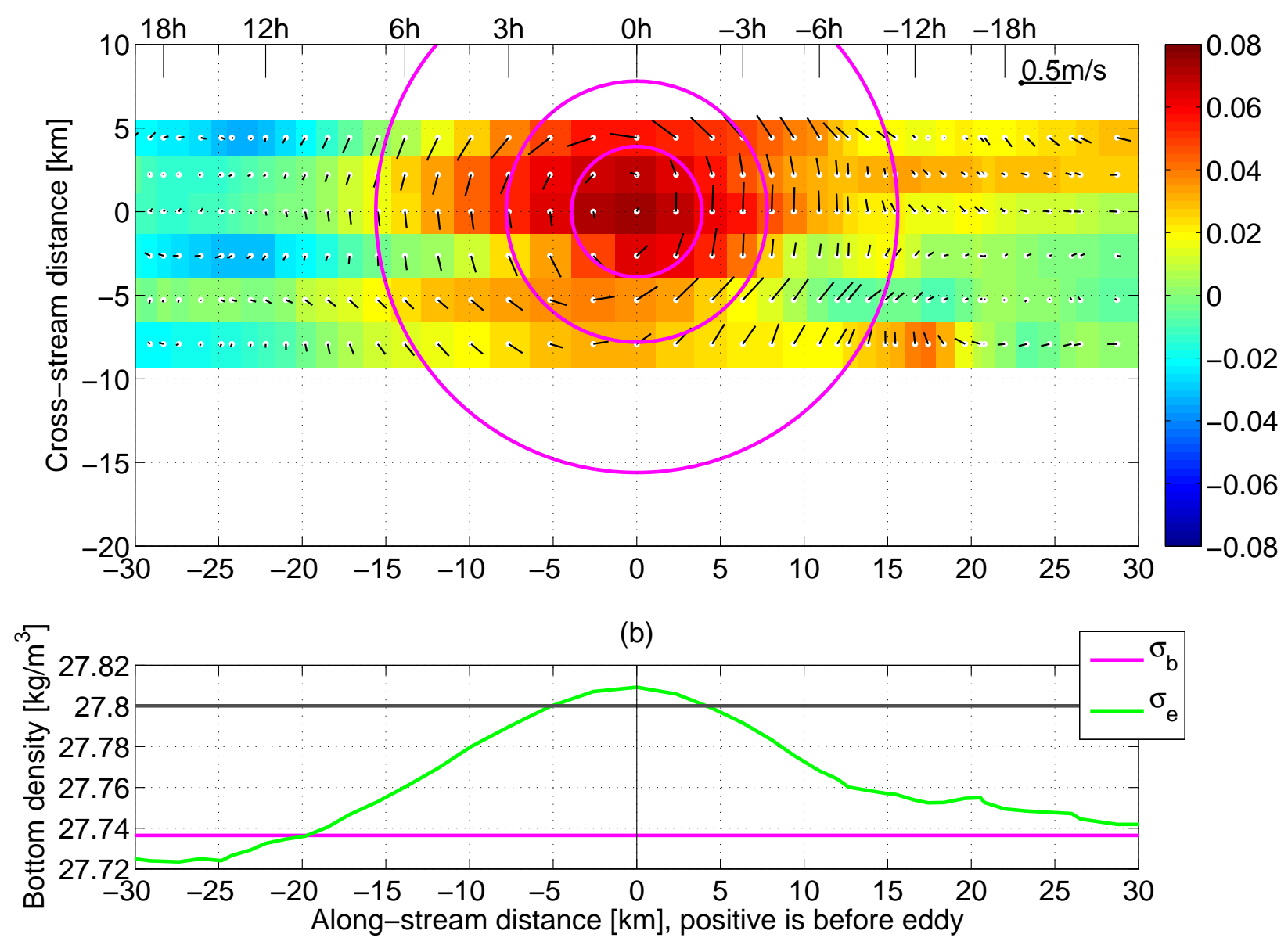

Figure 12: (a) Bottom density anomaly $\sigma_{e}^{\prime}$ (color) relative to the background bottom density of $27.735 \mathrm{~kg} / \mathrm{m}^{3}$. Velocity vectors are identical to Figure 10. (b) Background bottom density $\sigma_{b}$ and density $\sigma_{e}$ along center slice $(y=0)$ of cyclone. The classical DSOW definition of $27.8 \mathrm{~kg} / \mathrm{m}^{3}$ (Dickson and Brown, 1994) is highlighted. 
(a) Background velocity

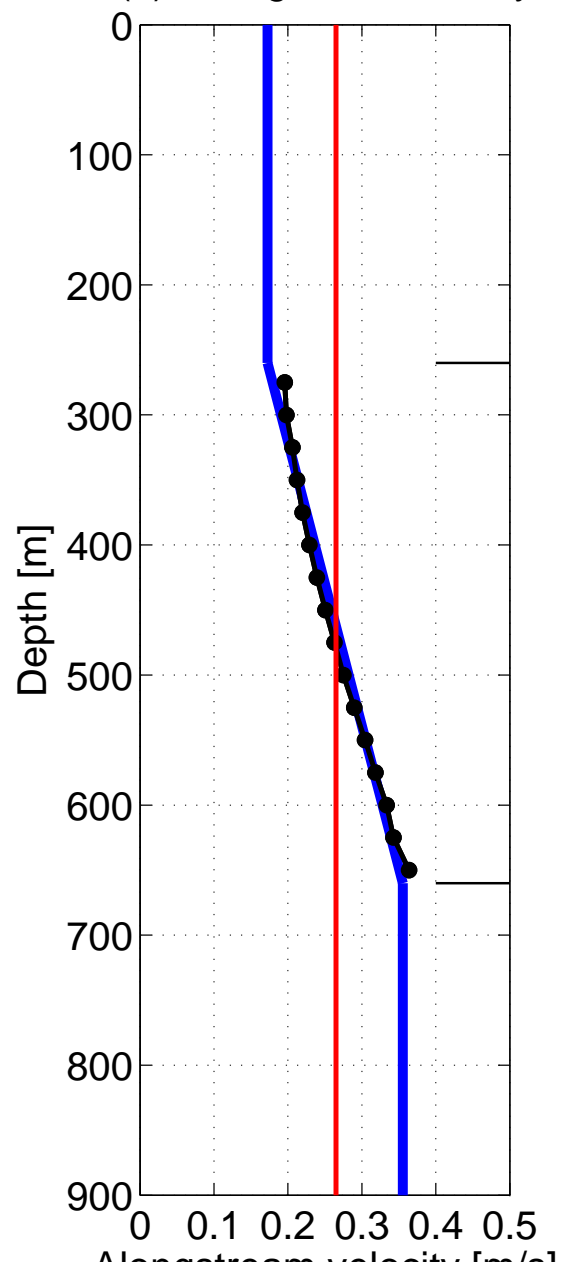

(b) Azimuthal velocity

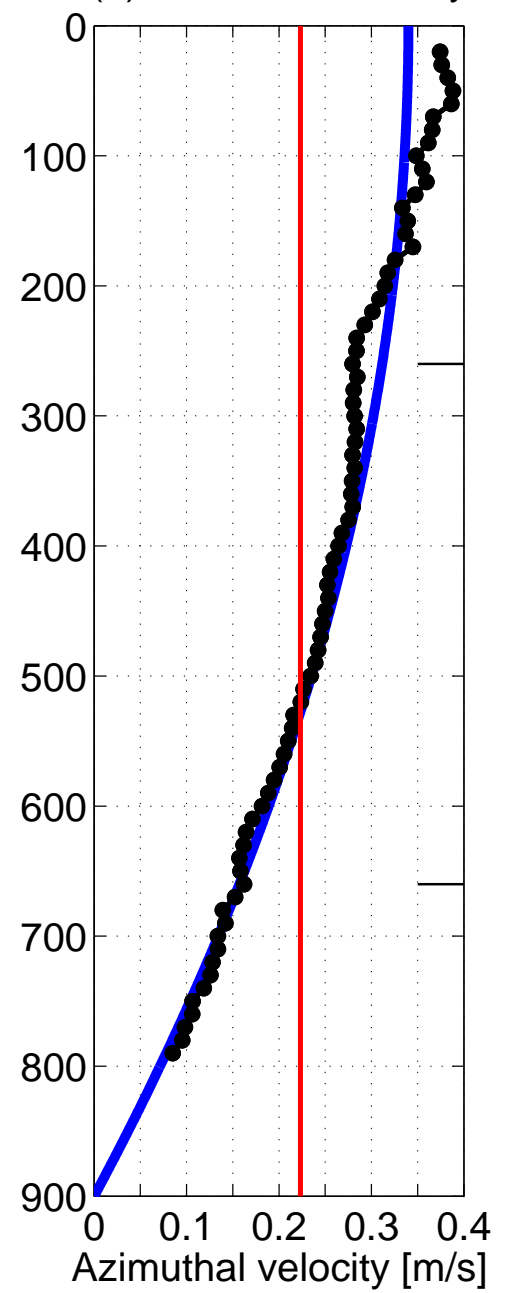

(c) Background density

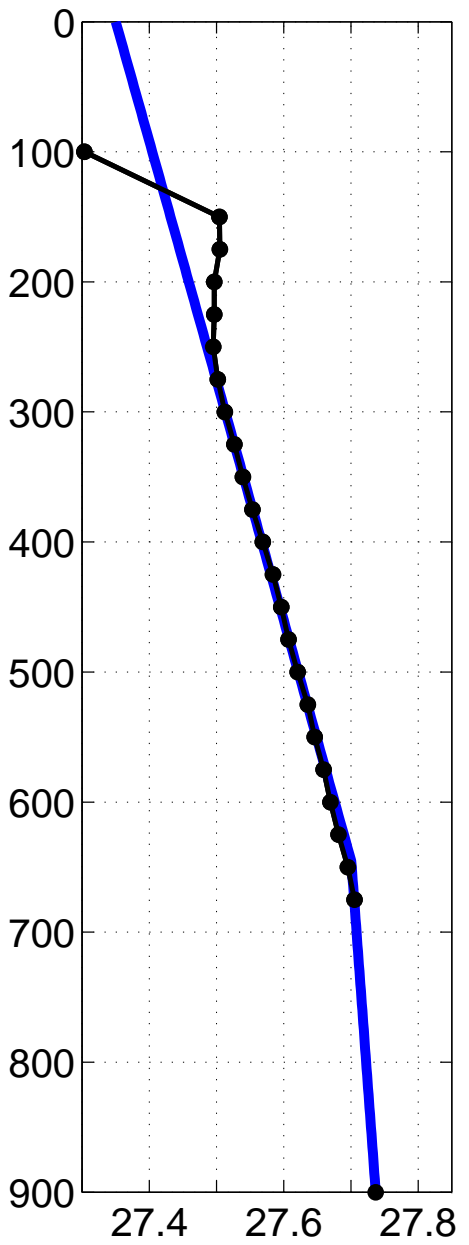

Potential density $\left[\mathrm{kg} / \mathrm{m}^{3}\right]$

Figure 13: (a) Vertical structure of the background alongstream velocity at EG4. The fit (blue) is linear where constrained by data and constant elsewhere. The depth-mean (red) is also shown. (b) Vertical structure of the azimuthal velocity $v_{0}$ as defined in Equation (4). The fit is quadratic. (c) Vertical structure of the background potential density. The fit is piecewise linear above and below $650 \mathrm{~m}$. 
(a) $\left(u-u_{b}-u_{b}{ }^{\prime}\right)$ : Eddy associated alongstream velocity $[\mathrm{m} / \mathrm{s}]$

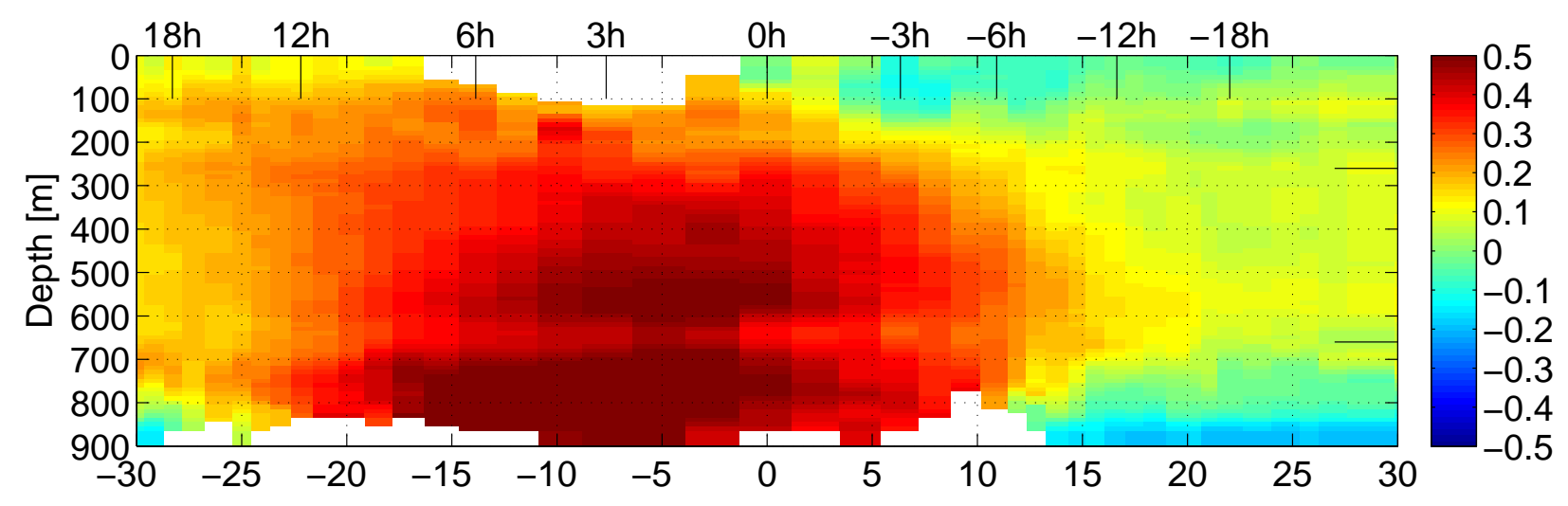

(b) $\left(\mathrm{v}-\mathrm{v}_{\mathrm{b}}\right)$ : Eddy associated cross-stream velocity $[\mathrm{m} / \mathrm{s}]$

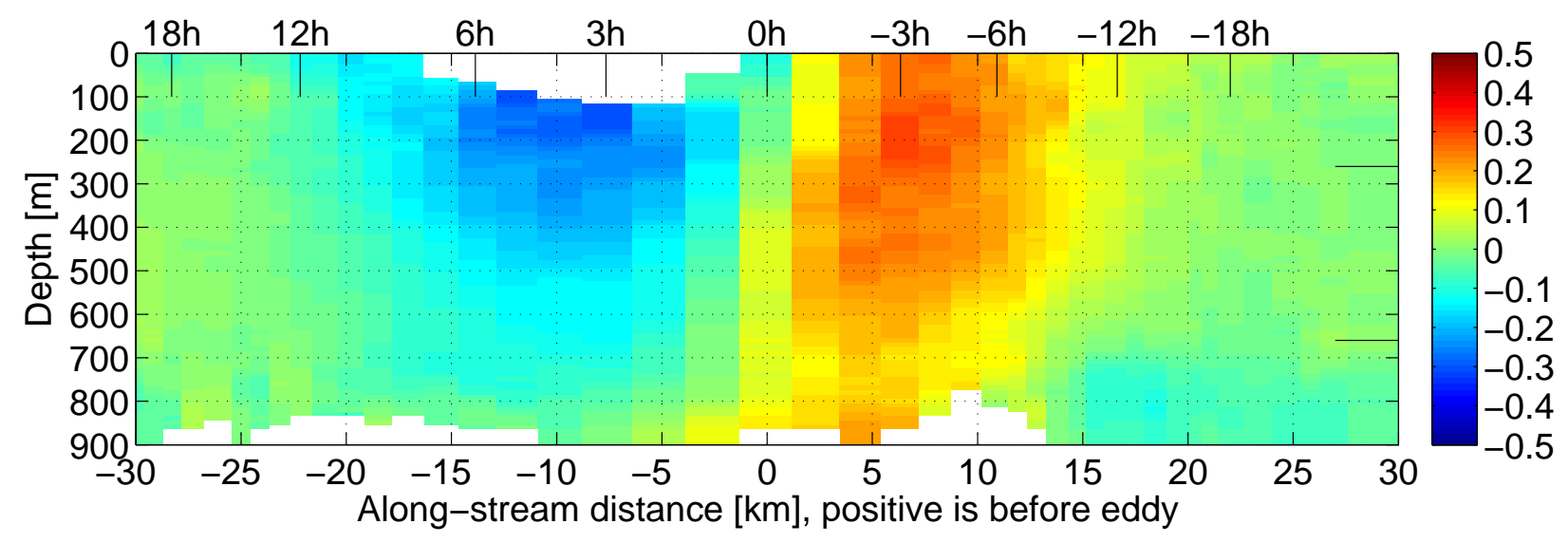

Figure 14: Alongstream section of the eddy associated velocity field of the composite eddy $\left(u-u_{b}-u_{b}^{\prime}(z), v-\right.$ $\left.v_{b}\right)$. The velocities have been averaged in the cross-stream direction from $y=-6 \mathrm{~km}$ to $y=6 \mathrm{~km}$. 
Time w.r.t. to eddy center passage [h], negative is before eddy

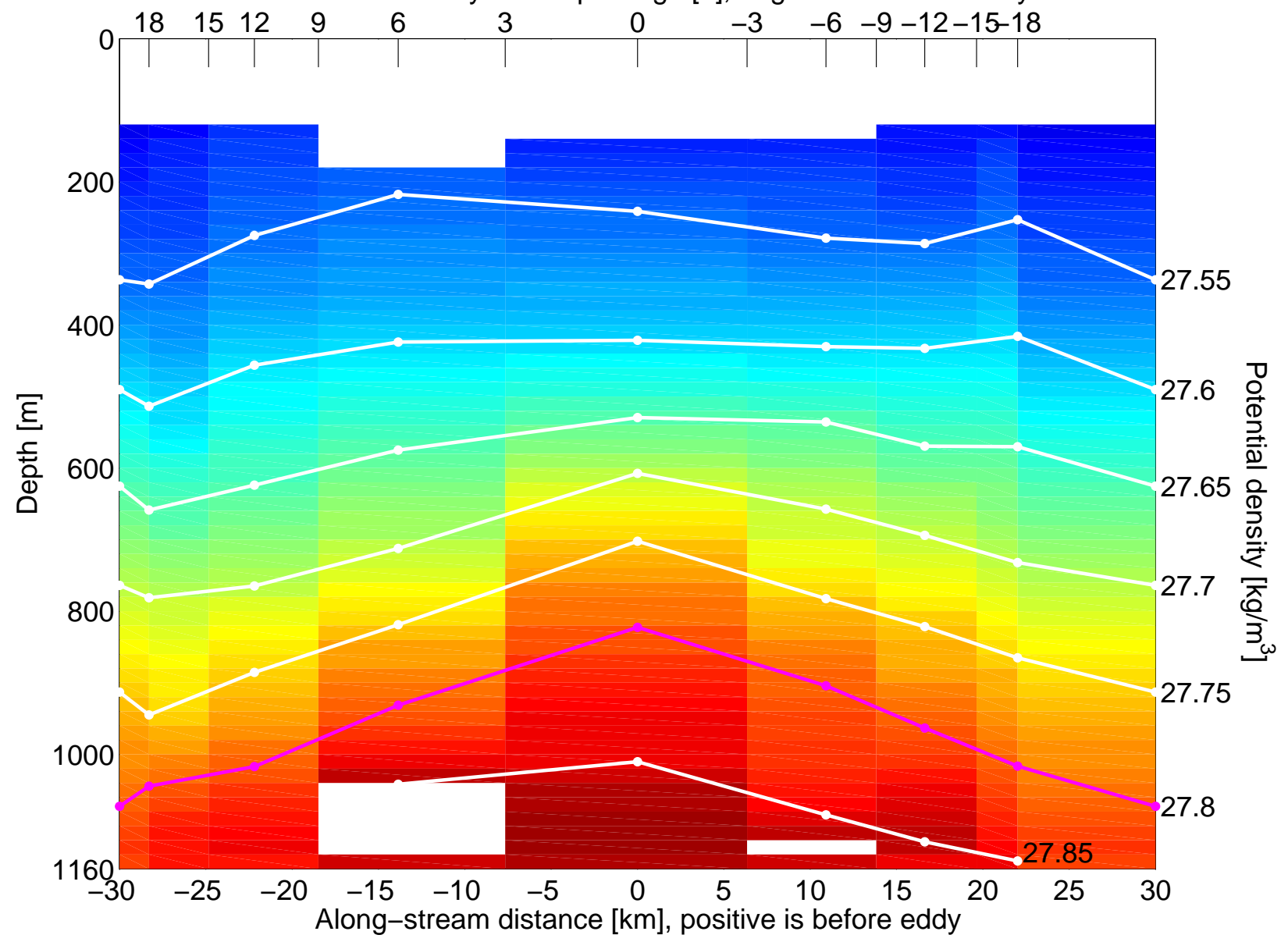

Figure 15: Mean alongstream section of potential density at EG5 during the passage of 66 cyclones near and onshore of EG4. The horizontal offset from the cyclone centers is $7-10 \mathrm{~km}$. The densities of all available profiles were binned ( 6 hours temporally, $20 \mathrm{~m}$ vertically) and averaged. The bin averages are shown in color (bins containing less than 6 individual profiles are not shown). Lines (magenta for 27.8, white for others) track the height of the isopycnals between temporal bin centers. 
(a) Azimuthal velocity [m/s]

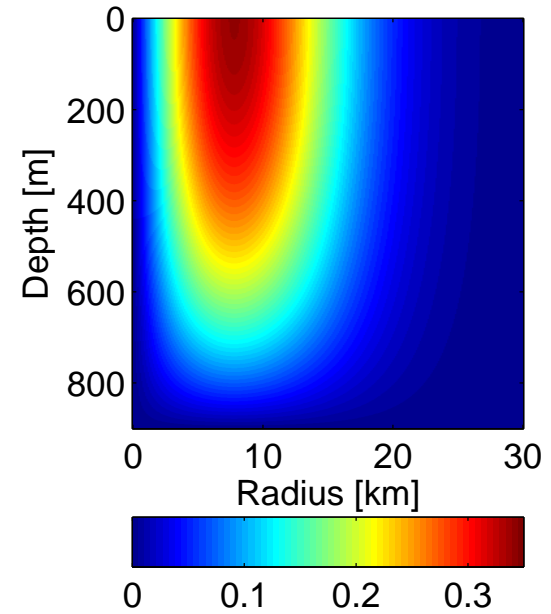

(d) Bottom dens anomaly $\left[\mathrm{kg} / \mathrm{m}^{3}\right]$ (b) Dynamic pressure $[\mathrm{Pa}]$

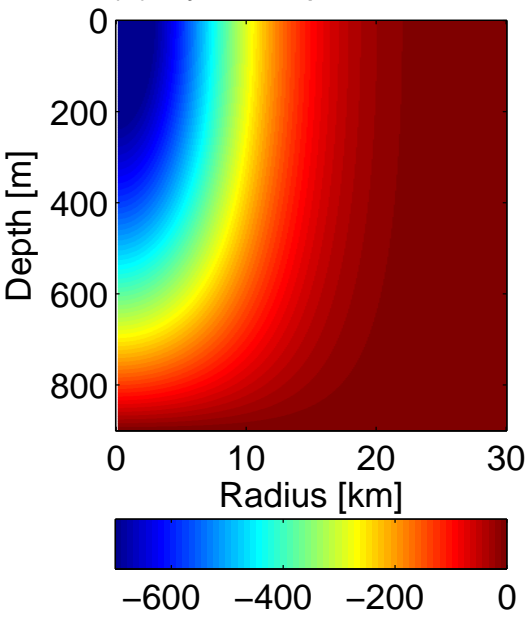

(e) Density based on pres grad (c) Density anomaly $\left[\mathrm{kg} / \mathrm{m}^{3}\right]$

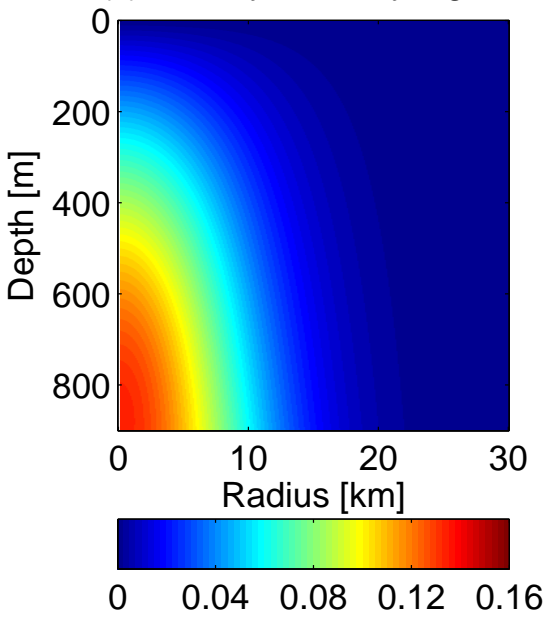

(f) Density based on microcat

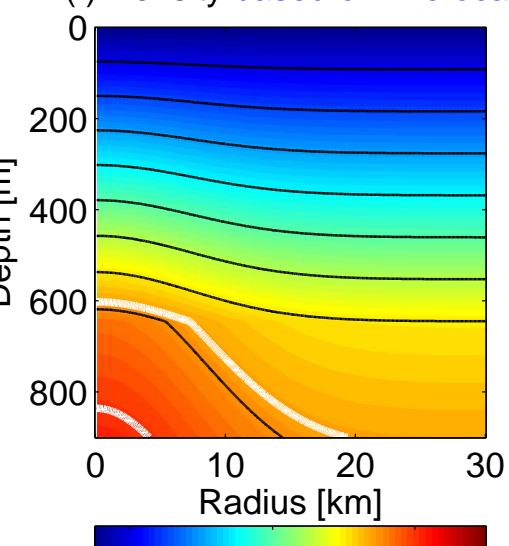

27.4

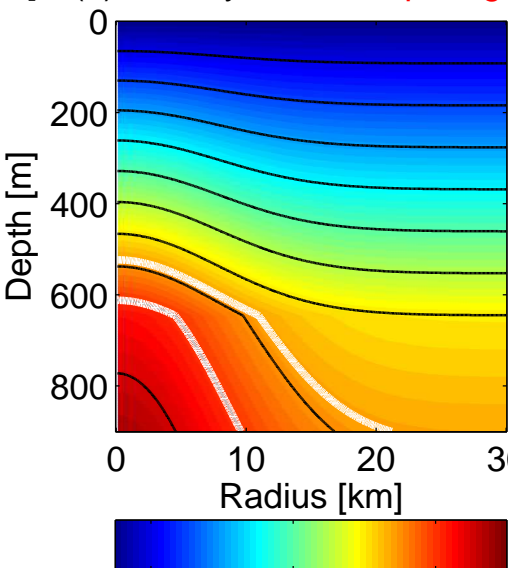

27.4

27.6

27.8

Figure 16: Constructed fields for the calculation of the density field inside smooth fitted DSOW cyclones in the radius-depth plane (see text for methodology). (a) Azimuthal velocity, (b) Dynamic pressure from the horizontal integral of the velocity field, (c) Density anomaly from the vertical derivative of the pressure field, (d) Bottom density anomaly from the pressure gradient calculation shown in (c) and from the fit to the bottom microcat data (Figure 11b); the density anomaly corresponding to a total density of 27.8 is also shown, (e,f) Estimates of the total density $\left[\mathrm{kg} / \mathrm{m}^{3}\right]$ which is the sum of the background profile in Figure 13c and the density anomaly in (c); the 27.8 and 27.74 isopycnals are contoured in white, (e) is an upper estimate using the anomaly field in (c), (f) is a lower estimate using the anomaly field in (c) divided by 1.8 which is the ratio of the two amplitudes in $(\mathrm{d})$. 


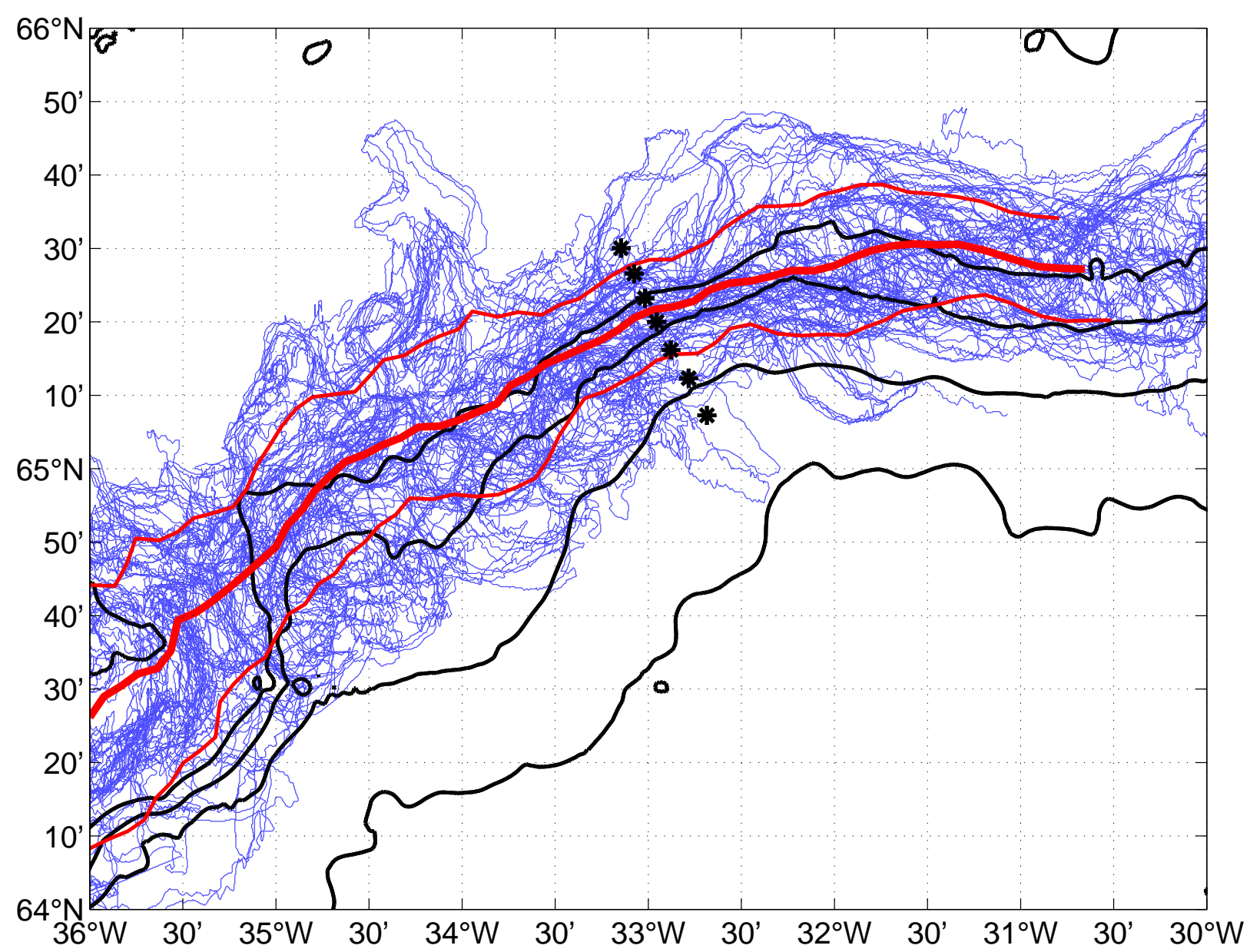

Figure 17: Location of the $6^{\circ} \mathrm{C}$ sea surface temperature isotherm indicating the East Greenland/Irminger Current front. The 63 individual realizations (blue) during September 2007 to October 2008 and their mean \pm 1 standard deviation (red) are shown as are the $(500,1000,1500,2000) \mathrm{m}$ isobaths and the mooring locations (black stars). 


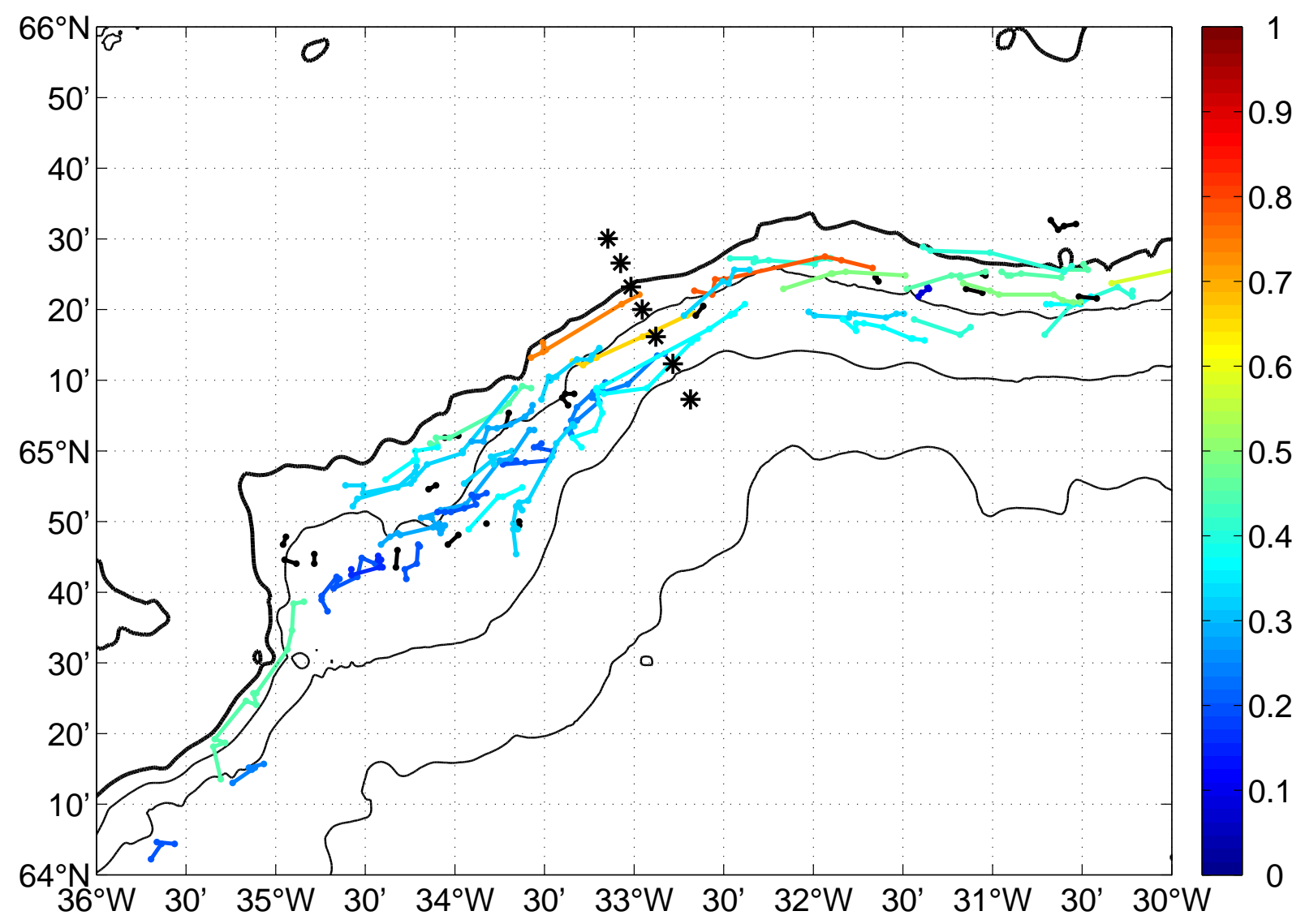

Figure 18: Propagation of the 40 SST disturbances that were trackable (speed $[\mathrm{m} / \mathrm{s}]$ is shown in color). Non-trackable disturbances are shown in black. Each dot refers to an observation of the center of a SST disturbance from a partially cloud free SST image; the time between consecutive dots is not systematic. 
Table 1: Summary of DSOW cyclone statistics. The statistics of the SST disturbances described in Bruce (1995) are contrasted with the DSOW cyclone values obtained from the subsurface mooring array in this study.

\begin{tabular}{|c|c|c|c|c|c|}
\hline & & \multicolumn{2}{|c|}{ Bruce (1995) } & \multicolumn{2}{|r|}{ this study } \\
\hline Variable & Name & Mean & Method & Mean & Method \\
\hline$u_{t}+u_{b}$ & $\begin{array}{c}\text { Translational } \\
\text { speed }\end{array}$ & $\begin{array}{l}0.27 \mathrm{~m} / \mathrm{s} \\
\pm 0.11 \mathrm{~m} / \mathrm{s}\end{array}$ & $\begin{array}{l}\mathrm{n}=33, \text { feature } \\
\text { tracking in SST }\end{array}$ & $0.72 \mathrm{~m} / \mathrm{s}$ & $\begin{array}{c}\mathrm{n}=101, \text { composite } \\
\text { eddy velocities }\end{array}$ \\
\hline$R_{0}$ & Radius & $17 \mathrm{~km}$ & $\begin{array}{c}\mathrm{n}=46, \text { spiral } \\
\text { dimensions in } \mathrm{SST}\end{array}$ & $7.8 \mathrm{~km}$ & $\begin{array}{c}\mathrm{n}=101, \text { composite } \\
\text { eddy velocities }\end{array}$ \\
\hline$v_{a}$ & $\begin{array}{c}\text { Peak azimuthal } \\
\text { velocity }\end{array}$ & & & $0.22 \mathrm{~m} / \mathrm{s}$ & $\begin{array}{c}\mathrm{n}=101, \text { composite } \\
\text { eddy velocities }\end{array}$ \\
\hline$D$ & $\begin{array}{c}\text { Distance } \\
\text { between features }\end{array}$ & $54 \mathrm{~km}$ & $\begin{array}{c}\mathrm{n}=54, \text { features in } \\
\text { same SST image }\end{array}$ & $130 \mathrm{~km}$ & $D=\left(u_{t}+u_{b}\right) * T$ \\
\hline$T$ & $\begin{array}{c}\text { Time } \\
\text { between features }\end{array}$ & 2.3 days & $T=D /\left(u_{t}+u_{b}\right)$ & 2.1 days & $\begin{array}{c}\mathrm{n}=190, \text { eddy } \\
\text { center identification }\end{array}$ \\
\hline$\alpha^{\prime}$ & $\begin{array}{c}\text { Feature } \\
\text { descent rate }\end{array}$ & $2.3 \mathrm{~m} / \mathrm{km}$ & $\begin{array}{c}\mathrm{n}=35, \text { center } \\
\text { locations along slope }\end{array}$ & $2.7 \mathrm{~m} / \mathrm{km}$ & $\alpha^{\prime}=\frac{v_{b}}{u_{t}+u_{b}} \frac{d H}{d y}$ \\
\hline
\end{tabular}

